Avaliação da proficiência em inglês acadêmico através de um teste adaptativo informatizado 


\title{
Avaliação da proficiência em inglês acadêmico através de um teste adaptativo informatizado
}

\author{
Vanessa Rufino da Silva
}

Orientadora: Profa. Dra. Mariana Cúri

\begin{abstract}
Dissertação apresentada ao Instituto de Ciências Matemáticas e de Computação - ICMC-USP e ao Departamento de Estatística - DEs-UFSCar, como parte dos requisitos para obtenção do título de Mestre em Estatística - Interinstitucional de Pós-Graduação em Estatística. EXEMPLAR DE DEFESA
\end{abstract}


Ficha catalográfica elaborada pela Biblioteca Prof. Achille Bassi e Seção Técnica de Informática, ICMC/USP, com os dados fornecidos pelo(a) autor(a)

Rufino da Silva, Vanessa
Avaliação da proficiência em inglês acadêmico
através de um teste adaptativo informatizado /
Vanessa Rufino da Silva; orientadora Mariana Cúri. -
- São Carlos, 2015.
33 p.
Dissertação (Mestrado - Programa
Interinstitucional de Pós-graduação em Estatística) --
Instituto de Ciências Matemáticas e de Computação,
Universidade de São Paulo, 2015.
1. Teste Adaptativo Informatizado. 2. Exame de
Proficiência em Inglês. 3. Teoria de Resposta ao
Item 4 Shadow test. I. Cúri, Mariana, orient.
II. Título.




\section{Resumo}

Este trabalho descreve as etapas de transformação de um exame de proficiência em inglês acadêmico, aplicado via lápis-e-papel, com itens de múltipla escolha administrados segundo o método de Medida de Probabilidade Admissível (Shuford Jr et al, 1966), utilizado no programa de pósgraduação do Instituto de Ciências Matemáticas e de Computação da Universidade de São Paulo (ICMC-USP), em um teste adaptativo informatizado (TAI-PI) baseado em um modelo da Teoria de Resposta ao Item (TRI). Apesar do programa aceitar diversos exames que atestam a proficiência em inglês para indivíduos não-nativos de abrangência e reconhecimento internacionais, como o TOEFL (Test of English as a Foreign Language), IELTS (International English Language Testing System) e CPE (Certificate of Proficiency in English), por exemplo, a sua obrigatoriedade é incoerente em universidades públicas do Brasil devido ao custo que varia de 200 a 300 dólares por exame. O software TAI-PI (Teste Adaptativo Informatizado para Proficiência em Inglês), que foi desenvolvido em Java e SQLite, será utilizado para a avaliação da proficiência em inglês dos alunos do programa a partir do segundo semestre de 2013, de forma gratuita. A metodologia estatística implementada foi definida considerando a história e objetivos do exame e adotou o modelo de resposta gradual unidimensional de Samejima (Samejimą 1969), o critério de Kullback-Leibler para seleção de itens, o método de estimação da esperança a posteriori para os traços latentes (Baker, 2001) e a abordagem Shadow test (Van der Linden e Pashley 2010) para imposição de restrições (de conteúdo e tamanho da prova) na composição do teste de cada indivíduo. Uma descrição da estrutura do exame, dos métodos empregados, dos resultados das aplicações do TAI-PI a alunos de pós-graduação do ICMC e estudos de classificação dos alunos em aprovados e reprovados, são apresentados neste trabalho, evidenciando a boa qualidade da nova proposta adotada e aprimoramento do exame com a utilização dos métodos de TRI e TAI.

Palavras-chave: teste adaptativo computadorizado, teoria de resposta ao item, shadow test. 


\section{Abstract}

This work describes the steps for converting a linear paper-and-pencil English proficiency test for academic purposes, composed with multiple choice items that are administered following the admissible probability measurement procedure (Shuford Jr et al, 1966), adopted by the graduate program of Institute of Mathematical Sciences and Computing of University of São Paulo (ICMCUSP), Brazil, to a computerized adaptive test (TAI-PI) based on an item response theory model (IRT). Despite the Institute recognizes reliable international English-language exams for academic purposes and non-native speakers, as TOEFL (Test of English as a Foreign Language), IELTS (International English Language Testing System) and CPE (Cambridge English: Proficiency), for instance, it is inconsistent that public universities in Brazil require them as certification because of the cost of approximately US\$200.00 to US $\$ 300.00$ per exam. The software TAI-PI (computerized adaptive test for English proficiency) was implemented in Java language, used SQLite as database engine, and it shall be offered free of charge for English proficiency assessment of the graduate students from October 2013. The statistical methodology employed for TAI-PI construction was defined considering the history and the aims of the evaluation and adopted the Samejima's graded response model (Samejima, 1969), the Kullback-Leibler information criterion for item selection, the expected a posteriori Bayesian estimation for latent trait (Baker 2001) and shadow test approach (Van der Linden e Pashley, 2010) for test constraints (content and size of the test, for example). A description of the test design, the employed statistical methods, study results of a real application of TAI-PI to graduate students are presented in this work and the validation studies of the new methodology for pass/fail classification, highlighting the good quality of the new evaluation system and examination of improvement with the use of the methods of IRT and CAT.

Keywords: computerized adaptive test, item response theory, shadow test. 


\section{Sumário}

Lista de Abreviaturas vii

$\begin{array}{lc}\text { Lista de Figuras } & \text { ix }\end{array}$

Lista de Tabelas $\quad$ xi

1 Introdução $\quad 1$

2 Metodologia TAI-PI $\quad 5$

2.1 Número de categorias $\ldots \ldots \ldots \ldots \ldots \ldots \ldots$

2.2 Modelo de Samejima . . . . . . . . . . . . . . . . . . . . . 7

2.3 Estimação do traço latente . . . . . . . . . . . . . . . . 9

2.4 Seleção de itens . . . . . . . . . . . . . . . . . . . . . . 9

2.5 Shadow test . . . . . . . . . . . . . . . . . . . . . 10

2.6 Critério de início e parada . . . . . . . . . . . . . . . . . . . 11

$\begin{array}{lll}3 & \text { Estudo de simulação } & 13\end{array}$

4 Aplicação real $\quad 17$

5 Estudos de classificação $\quad 25$

6 Conclusões $\quad 29$

6.1 Sugestões para Pesquisas Futuras . . . . . . . . . . . . . . . . . . . . . 29

$\begin{array}{ll}\text { Referências Bibliográficas } & 31\end{array}$ 


\section{Lista de Abreviaturas}

$\begin{array}{ll}\text { TOEFL } & \text { Test of English as a Foreign Language } \\ \text { IELTS } & \text { International English Language Testing System } \\ \text { CPE } & \text { Certificate of Proficiency in English } \\ \text { CCMC } & \text { Ciências da Computação e Matemática Computacional } \\ \text { PIPGEs } & \text { Programa Interinstitucional de Pós Graduação em Estatística } \\ \text { ICMC } & \text { Instituto de Ciências Matemáticas e de Computação } \\ \text { USP } & \text { Universidade de São Paulo } \\ \text { UFSCar } & \text { Universidade Federal de São Carlos } \\ \text { EPI } & \text { Exame de Proficiência em Ingçês } \\ \text { TAI } & \text { Teste Adaptativo Informatizado } \\ \text { TRI } & \text { Teoria de Resposta ao Item } \\ \text { GRE } & \text { Graduate Record Examination } \\ \text { ETS } & \text { Educational Testing Service } \\ \text { ASBAV } & \text { Armed Services Vocational Aptitude Test Battery } \\ \text { TAI-PI } & \text { Teste Adaptativo Informatizado de Proficiência em Inglês } \\ \text { MPA } & \text { Medida de Probabilidade Admissível } \\ \text { CALEAP-WEB } & \text { Computer-Aided Learning of English for Academic Purposes } \\ \text { MRG } & \text { Modelo de Resposta Gradual } \\ \text { EAP } & \text { Esperança a Posteriori }\end{array}$




\section{Lista de Figuras}

1.1 Diagrama de um teste adaptativo informatizado . . . . . . . . . . . . 2

1.2 Exemplo de um item e sua possíveis alternativas de respostas no EPI. . . . . . . . . 3

1.3 Triângulo equilátero da MPA com a caracterização das alternativas de respostas de cada item da prova supondo a alternativa A como a correta . . . . . . . . . . . . 4

1.4 Critério de classificação com base em MPA . . . . . . . . . . . . . . . . . . . 4

2.1 Curva Característica do Item 25 com as 6 categorias iniciais . . . . . . . . . . . . 6

2.2 Curva Característica do Item 25 com agrupamento em 3 categorias . . . . . . . . . 6

2.3 Agrupamento das categorias de resposta aos itens adotado para o TAI-PI. . . . . . . 7

2.4 Exemplo de uma curva de probabilidade acumulada sob o modelo de Samejima com

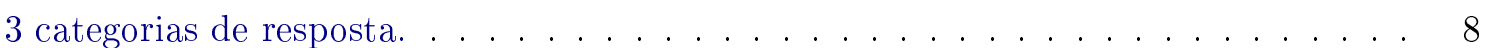

2.5 Exemplo de uma curva de probabilidade de escolha da categoria sob o modelo de Samejima com 3 categorias de resposta. . . . . . . . . . . . . . . . 8

2.6 Representaçao do Shadow test. Fonte: van der Linden, 2010 . . . . . . . . . . . . . . . 11

3.1 Valor verdadeiro em função do valor estimado de $\theta$ por 3 prioris, para diferentes tamanhos de teste $(20,25$ e 30 itens $) \ldots \ldots \ldots \ldots \ldots \ldots$

3.2 Vício de 1000 respondentes simulados estimado por 3 prioris, para diferentes tamanhos de teste $(20,25$ e 30 itens $) \ldots \ldots \ldots \ldots \ldots \ldots$

4.1 Boxplot da proficiência estimada após 25 e 40 itens respondidos dos 59 alunos na aplicação do TAI-PI, separadamente para o grupo reprovado e aprovado pelo critério MPA.

4.2 Proficiências estimadas do aluno 17 em cada passo do TAI-PI, a linha vermelha representa a estimativa final após 40 itens. . . . . . . . . . . . . . . . . . . 21

4.3 Proficiências estimadas do aluno 26 em cada passo do TAI-PI, a linha vermelha representa a estimativa final após 40 itens. . . . . . . . . . . . . . . . . . 22

4.4 Proficiências estimadas do aluno 46 em cada passo do TAI-PI, a linha vermelha representa a estimativa final após 40 itens. . . . . . . . . . . . . . . . . 23

5.1 Curva ROC para a proficiência de acordo com a classificação MPA . . . . . . . . . 25

5.2 Densidades estimadas para a proficiência por grupo . . . . . . . . . . . . 26 


\section{Lista de Tabelas}

3.1 Vício dos estimadores para diferentes prioris. . . . . . . . . . . . . . . . . . 14

3.2 Erro Quadrático Médio dos estimadores para diferentes prioris. . . . . . . . . . . . 14

4.1 Banco de itens do TAI-PI, maio de $2014 \ldots \ldots \ldots \ldots \ldots \ldots$

4.2 Estimativas dos traços latentes (após 25 e 40 itens respondidos) e classificação pelo MPA na aplicaçao do TAI- de maio de 2014 . . . . . . . . . . . . . . . . . 19

4.3 Porcentagem de acertos de 4 alunos em cada categoria na aplicação de maio de 2014. 20

5.1 Acurácia para o ponto de corte no estudo de simualção . . . . . . . . . . . . . . . . 27 


\section{Capítulo 1}

\section{Introdução}

O domínio da língua inglesa por alunos de pós-graduação em diversas áreas da ciência é fundamental, tanto para uma adequada absorção do conteúdo do curso, quanto para o desenvolvimento e divulgação do trabalho de pesquisa realizado. Neste contexto, muitas instituições de pós-graduação no Brasil exigem uma comprovação do domínio do inglês por seus alunos, seja no ato de ingresso no programa, seja ao longo de seu curso.

Há diversos exames de abrangência e reconhecimento internacionais normalmente aceitos, que atestam a proficiência em inglês para indivíduos não-nativos. Dentre os mais tradicionais, podemos citar o Test of English as a Foreign Language (TOEFL), o International English Language Testing System (IELTS) e o Certificate of Proficiency in English (CPE). No entanto, um dos maiores inconvenientes destes exames é o custo, que varia de 200 a 300 dólares americanos, aproximadamente. Isto torna a sua obrigatoriedade incoerente em universidades públicas de nosso país. Essa é uma das principais razões dos programas de pós-graduação em Ciências da Computação e Matemátia Computacional (CCMC) e em Estatística (PIPGEs) do ICMC-USP, sendo este último em conjunto com a UFSCar, oferecerem um Exame de Proficiência em Inglês (EPI) como uma alternativa gratuita a seus alunos.

Até o ano de 2013, o EPI era aplicado no formato lápis-e-papel e este trabalho tem como objetivo torná-lo um teste adaptativo informatizado (TAI), no qual os itens são selecionados gradativamente para o indivíduo, de acordo com sua proficiência. O nível da proficiência do indivíduo é atualizado após cada resposta, com base em um modelo da Teoria de Resposta ao Item (TRI). Assim, itens muito fáceis ou muito difíceis para um determinado indivíduo nem chegam a ser apresentados a ele, diminuindo o tamanho do teste, seu tempo de realização e tornando-o mais eficiente, objetivo e com resultado imediato (Weiş̧ 1982). Na Figura 1.1 são apresentados os procedimentos de seleção e administração de itens no teste e atualização das estimativas das proficiências, iterativamente, até que algum critério de parada seja satisfeito. 


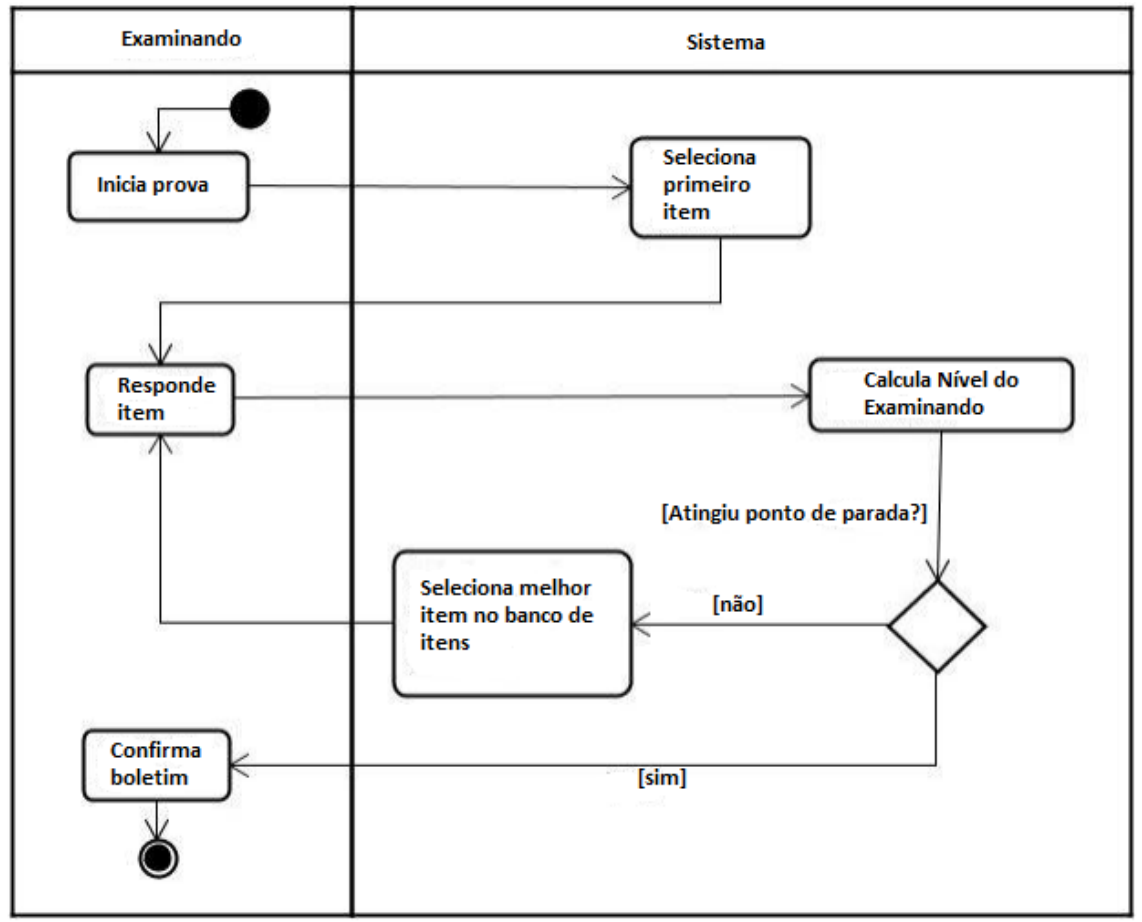

Figura 1.1: Diagrama de um teste adaptativo informatizado

Além das vantagens acima mencionadas, os testes adaptativos informatizados (TAI), em combinação com a TRI, possibilitam o cálculo de proficiências comparáveis entre indivíduos que responderam conjuntos diferentes de itens, e em momentos diferentes (Wainer et al, 2000). Isto facilita bastante a avaliação de construtos em larga escala, resultando no seu uso em importantes exames, tais como Graduate Record Examination (GRE) (Bridgeman e Clinę 2000; Eignor, 1993), desenvolvido pelo Educational Testing Service (ETS) em 1996; Test of English as a Foreign Language (TOEFL)(Eignor et al, 1998; Kirsch et al, 1998; Wainer e Wang 2000), também desenvolvido pelo ETS; Armed Services Vocational Aptitude Test Battery (ASBAV)(Sands et al, 1997; Segall 1993), desenvolvido pelo Departamento de Defesa dos Estados Unidos para selecionar potenciais recrutas para o serviço militar.

Para justificar a escolha da metodologia estatística adotada neste trabalho, apresentamos a seguir o formato com que o EPI foi aplicado no programa CCMC entre 2002 e 2013, servindo como um alicercee para a construção do Teste Adaptativo Informatizado de Proficiência em Inglês (TAI-PI).

Desde o ano 2002, o EPI consiste em uma prova composta por itens de múltipla escolha, aplicada semestralmente em formato lápis-e-papel. Para a confecção da prova, selecionam-se de 25 a 30 itens de um banco com 167 itens no total, subdivididos em 3 módulos da seguinte forma:

1. No módulo 1 são apresentados resumos de artigos de periódicos das áreas de Computação, Matemática Aplicada e Estatística e perguntas sobre seus componentes de estrutura científica.

2. No módulo 2 tem-se um trecho da introdução de um artigo científico, das mesmas áreas, e perguntas (em português) sobre compreensão do texto e relação entre ideias contidas nele.

3. No módulo 3 as perguntas estão relacionadas a convenções da língua inglesa, tais como conjunções, tempos verbais, relative clauses e artigos.

Os itens do banco foram elaborados, na época, por Andrea Jessica Borges Monzón, atual docente do Instituto Federal do Rio Grande do Sul, em conjunto com a professora Sandra Maria Aluísio, do Departamento de Computação do ICMC, sua orientadora de mestrado (Monzón 2010). A elaboração 
baseou-se na taxonomia de Bloom (Bloom 1984), objetivando avaliar a competência na leitura e compreensão de artigos científicos das áreas de Computação, Matemática Aplicada e Estatística.

Com o intuito de minimizar a possibilidade de "chute"da resposta correta e de não pressupor que o conhecimento do aluno sobre um determinado item é apenas binário (correto ou incorreto), a classificação da resposta do aluno segue a metodologia da Medida de Probabilidade Admissível (MPA) (Klinger 1997). A Figura 1.2 ilustra as possibilidades de resposta do aluno a um item do EPI. São três as alternativas de resposta em cada item de múltipla escolha (A, B e C), representadas nos vértices de um triângulo equilátero, sendo apenas uma delas a correta, no entanto, o aluno possui 13 possibilidades de resposta, que expressam o seu grau de certeza sobre qual das 3 afirmativas é a correta. Que podem ser selecionadas da maneira a seguir:

- Se estiver totalmente certo/seguro use as opções A, B ou C.

- Se estiver totalmente incerto/inseguro use a opção M.

- Se uma das opções A, B, C parece definitivamente errado, escolha entre as 5 opções da linha oposta.

- Indique preferência entre duas opções A, B ou C escolhendo as opções D, F, G, I, J ou L.

- Se duas opções A, B, ou C parecem iguais escolha as opções E, H ou K .

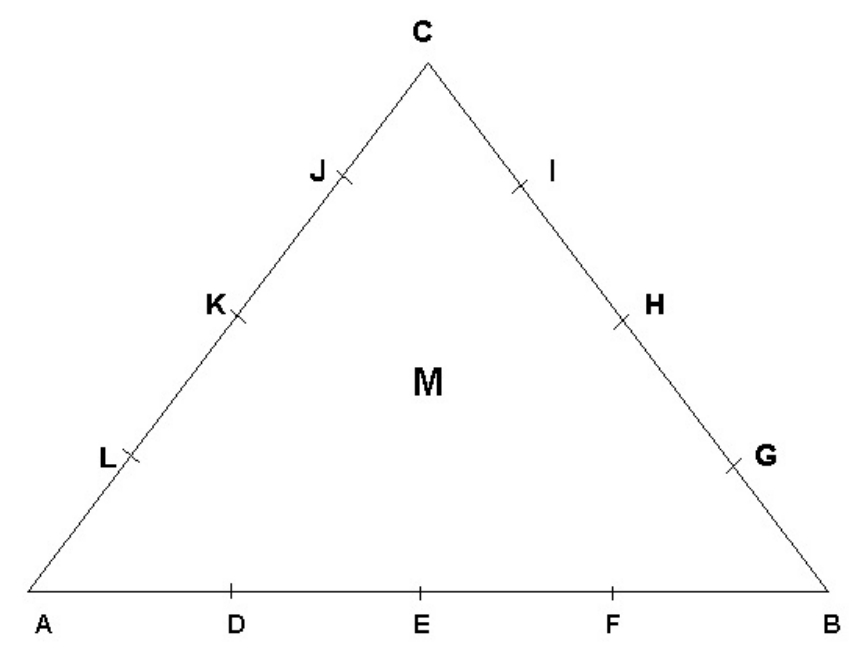

Questão 1: Quem é o Presidente dos Estados Unidos

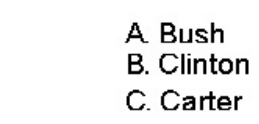

Ou Use as letras de Daté M

Figura 1.2: Exemplo de um item e sua possiveis alternativas de respostas no EPI.

Na Figura 1.3 tem-se a classificação da resposta do aluno de acordo com sua escolha em: totalmente informado, informado, parcialmente informado, mal informado, totalmente mal informado e não informado (Aluísio et al, 2003). 


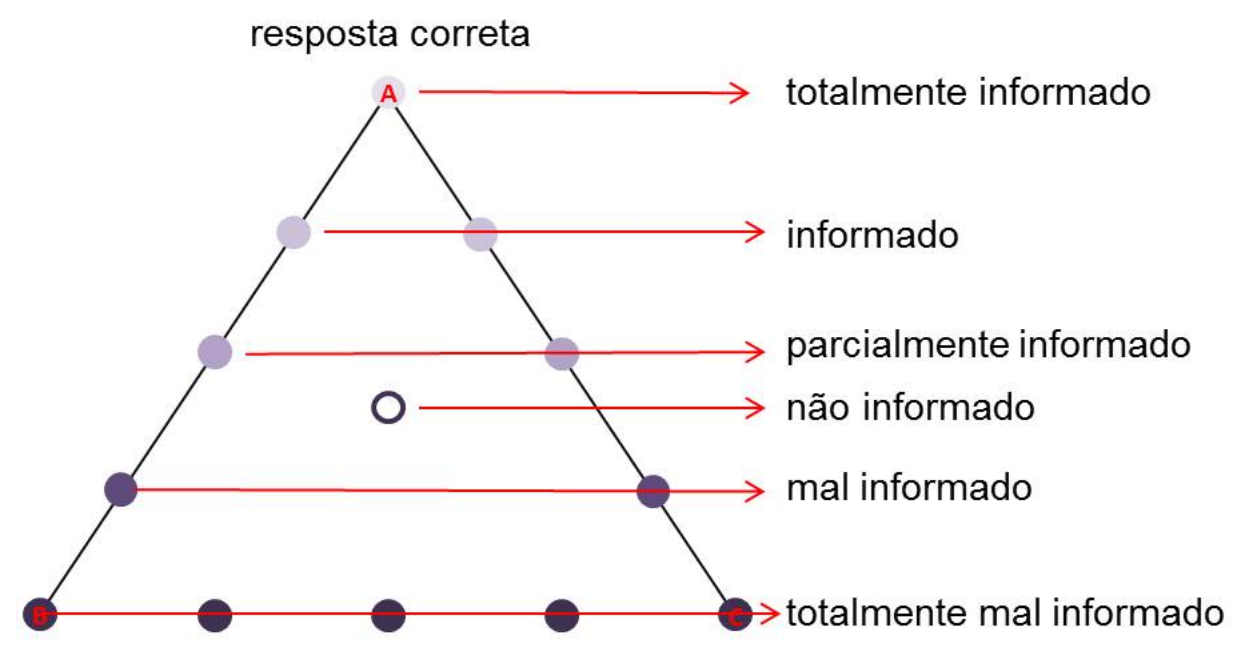

Figura 1.3: Triângulo equilátero da MPA com a caracterização das alternativas de respostas de cada item da prova supondo a alternativa A como a correta.

O aluno é aprovado se o percentual de respostas classificadas como "totalmente informado"for maior ou igual a $50 \%$ e se o percentual de respostas "totalmente mal informado"for menor ou igual a $25 \%$ ou se $90 \%$ ou mais de respostas estiverem nas classes "totalmente informado", "informado"e "parcialmente informado"e $10 \%$ ou menos de respostas na classe "totalmente mal informado", conforme Figura 1.4. Para os alunos não aprovados segundo esse critério, mas quase aprovados se não fossem as respostas de um ou dois itens (no máximo), um critério alternativo é disponibilizado considerando-se apenas o módulo 1,"Estrutura de Textos Científicos"(Figura 1.4).

\begin{tabular}{|c|c|c|c|}
\hline \multirow{2}{*}{ Pontuação } & \multicolumn{2}{|c|}{$\%$ de respostas } \\
\cline { 2 - 2 } & Critério 1 & Critério 2 & Módulo: Estrutura de Textos Científicos \\
\hline Totalmente Informado & $>=50 \%$ & \multirow{2}{*}{$>=90 \%$} & $>=50 \%$ \\
\hline Informado & & & $<2 \%$ \\
\hline Parcialmente Informado & & & \multirow{2}{*}{$<25 \%$} \\
\hline Mal Informado & & & \\
\hline Não Informado & & & \\
\hline Totalmente Mal Informado & $<25 \%$ & $<10 \%$ & \\
\hline
\end{tabular}

Figura 1.4: Critério de classificação com base em MPA.

Na perspectiva de auxiliar o estudo para o EPI, o aluno tem acesso a uma preparação orientada via web através de um ambiente computacional de aprendizado do inglês instrumental, denominado Computer-Aided Learning of English for Academic Purposes (CALEAP-Web). No ambiente, seu conhecimento de inglês é avaliado através de um teste e, com base nesse resultado, o aluno é orientado a realizar tarefas direcionadas a suprir as deficiências identificadas pelo teste anterior (Goncalves 2004; Piton-Gonçalves et al, 2009). 


\section{Capítulo 2}

\section{Metodologia TAI-PI}

O TAI-PI foi desenvolvido em Java 1.7.0 e SQLite versão 3.7.2 (Lanş 2006), para armazenamento do banco de dados.

O programa Same-CAT, criado por Thales Akira M. Ricarte ao longo de seu programa de mestrado, finalizado em 2012 pelo CCMC-ICMC, foi usado como base para a construção do TAIPI (Ricarte, 2013). O Same-CAT é um programa que permite a aplicação de testes adaptativos informatizados, com itens do banco calibrados de acordo com o modelo de resposta gradual de Samejima (1969) da TRI, critério de Kullback-Leibler para seleção de itens a cada passo do teste, estimação da proficiência pelo método bayesiano de esperança a posteriori e com abordagem Shadow test para imposição de restrições.

A versão atual do TAI-PI contou com a colaboração do aluno do Bacharelado em Ciências da Computação do ICMC, Leonardo Tadashi Myiake, para a inclusão de algumas facilidades na interação usuário-máquina.

Na Seção 2.1, apresentamos os estudos realizados para a definição de quantas categorias de respostas serão consideradas no modelo de Samejima. Na Seção 2.2, é definido o modelo de Samejima. Na Seção 2.3 descrevemos o método de estimação para as habilidades do TAI-PI. A Seção 2.4 traz o método de seleção de itens para o teste adaptativo. A Seção 2.5 apresenta o método Shadow Test para imposição de restrições dessa seleção. Finalmente, a Seção 2.6 descreve os critérios de início e parada do teste.

\subsection{Número de categorias}

As possibilidades de resposta a cada item do EPI podem ser consideradas ordinais com 13 possibilidades de respostas classificadas em 6 categorias de acordo com a Figura 1.3. Como o intuito deste trabalho é aprimorar o EPI mantendo e aproveitando o máximo possível sua história, o modelo de resposta gradual (MRG), proposto por Samejima (1969), foi adotado para o TAI-PI. No entanto, uma análise descritiva e inferencial sobre o número de categorias a ser considerado apontou para a necessidade de redução a 3 possiveis respostas em cada item.

Essa decisão foi baseada nas análises de uma parte dos 167 itens criados e aplicados no periodo de 2002 a 2012 no EPI formato lápis-e-papel, os quais foram filtrados 74 itens para a calibração dos parâmetros que tinham ao menos 80 respondentes ao longo do tempo (para obterem-se estimativas relativamente confiáveis dos parâmetros)e presentes em provas comuns (para garantir a equalização simultânea com a estimação dos parâmetros do modelo da TRI), no entanto devido ao pequeno tamanho amostral, algumas categorias de respostas não tinham frequências suficiente para estimar os parâmetros de itens.

Juntando isso com a análise exploratória das curvas característica de item (CCI), decidiu-se juntas as respostas em 3 categorias, Nas Figuras 2.1 e 2.2 são apresentados as CCI do item 25 antes e depois do agrupamento de categorias. 


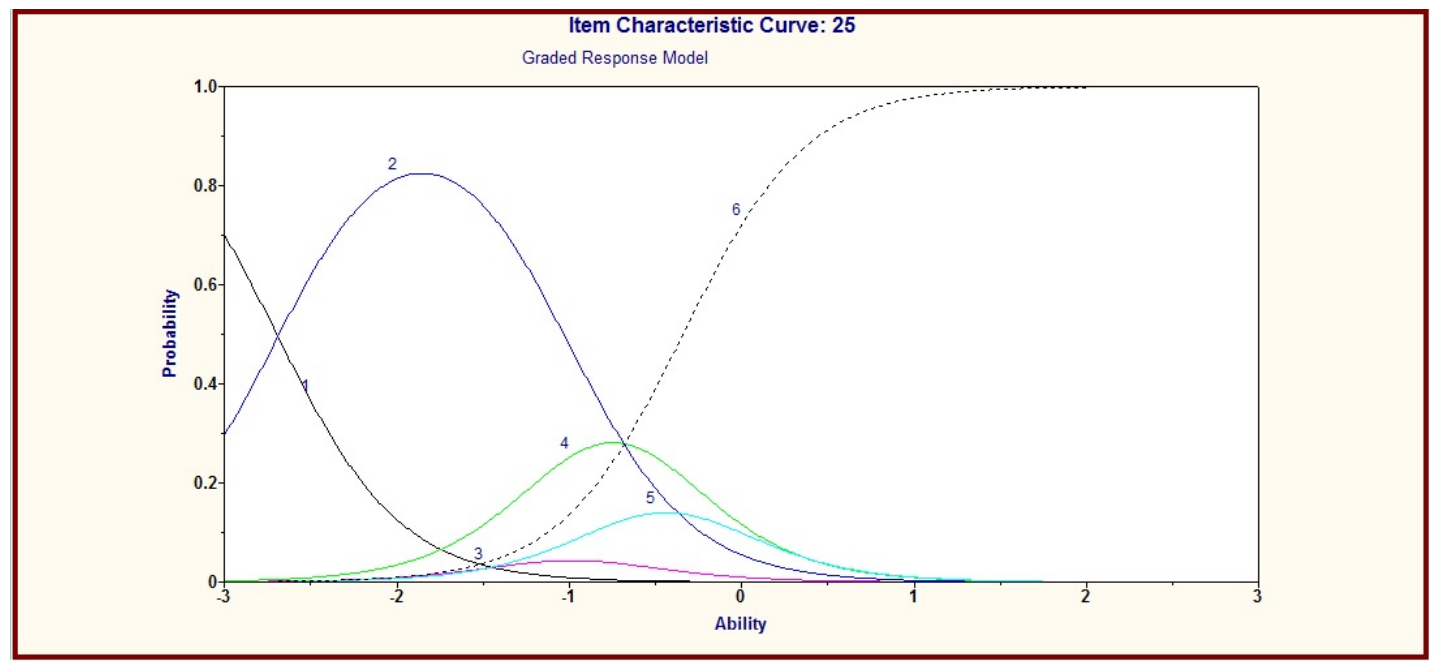

Figura 2.1: Curva Característica do Item 25 com as 6 categorias iniciais

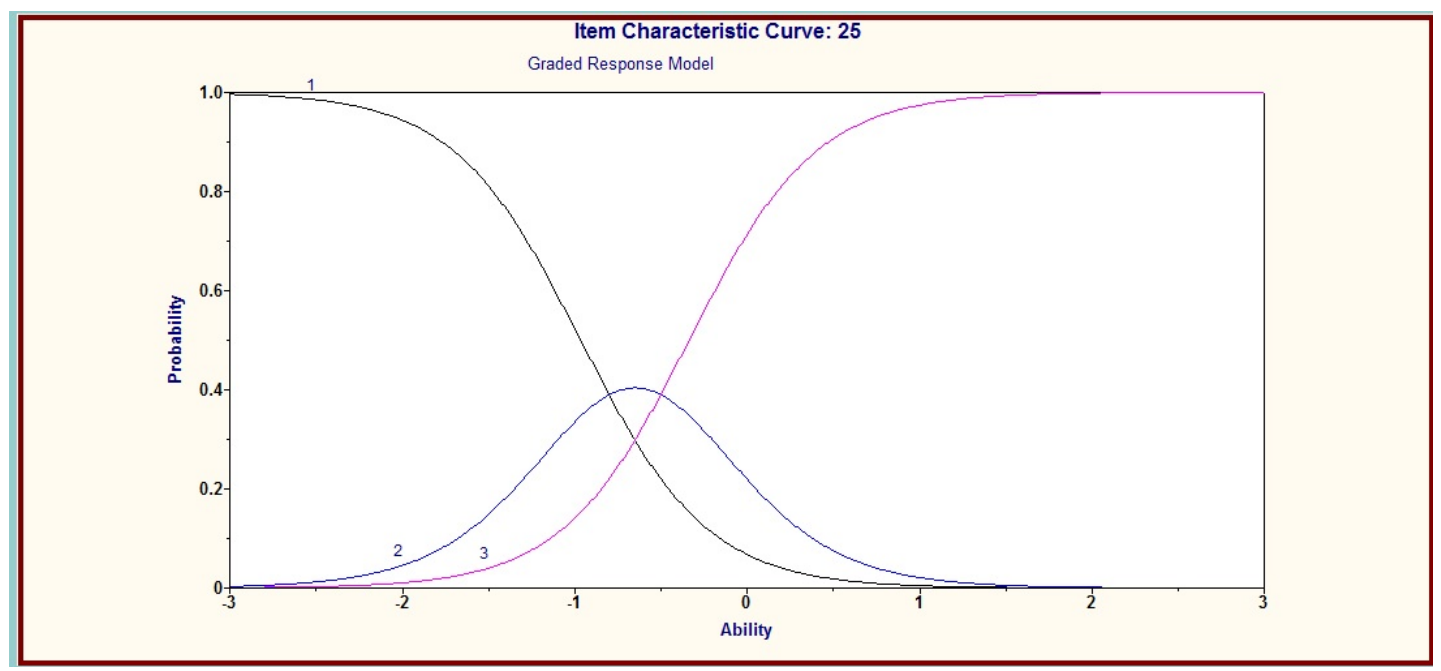

Figura 2.2: Curva Característica do Item 25 com agrupamento em 3 categorias

O agrupamento das categorias encontra-se definido na Figura 2.3. 


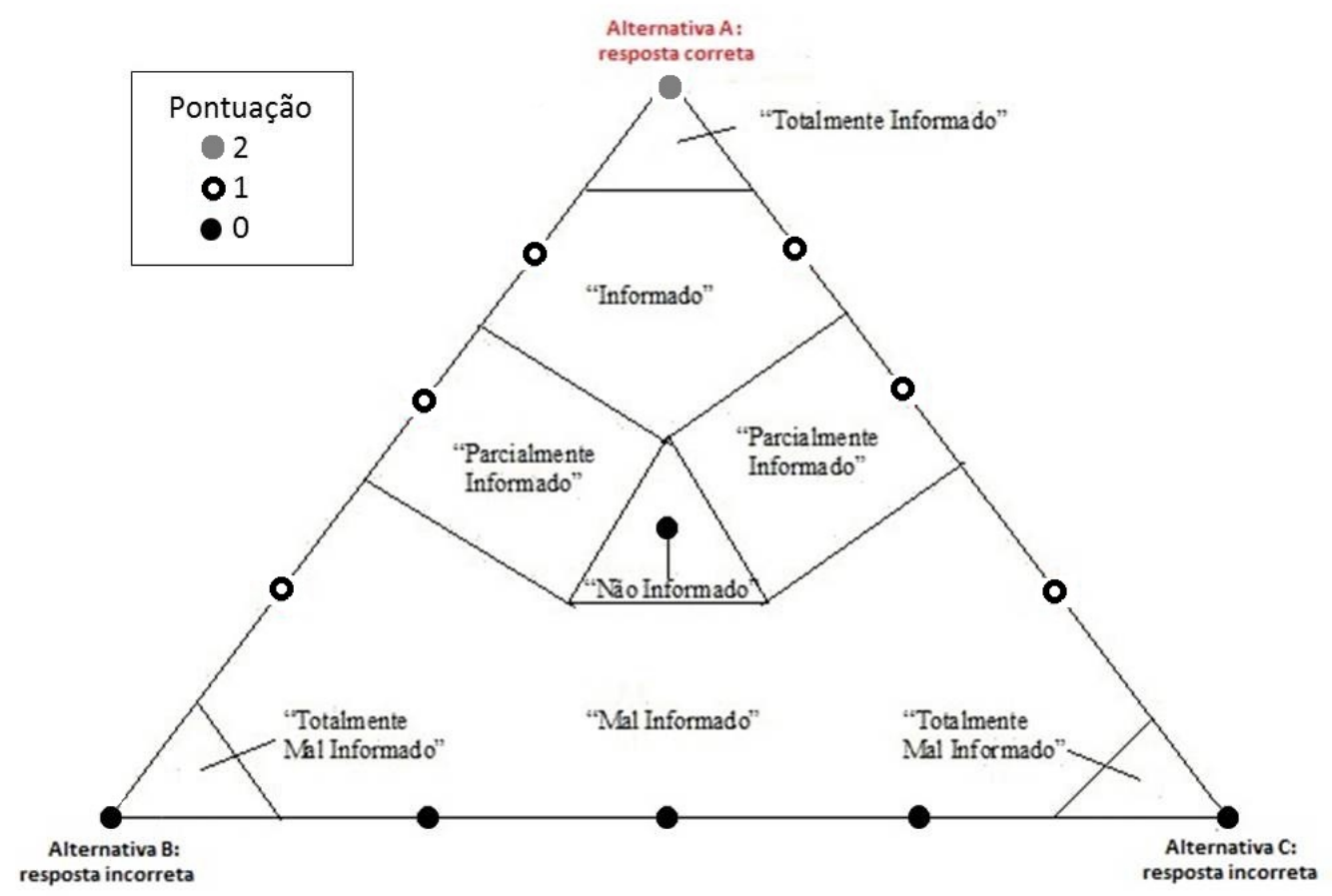

Figura 2.3: Agrupamento das categorias de resposta aos itens adotado para o TAI-PI.

\subsection{Modelo de Samejima}

Suponha $k=0,1,2$ denotanto as 3 categorias da Figura 2.3 categorias organizadas em ordem crescente, ou seja, quanto maior o valor de $k$, mais próxima ela estará da resposta totalmente correta. A probabilidade de um indivíduo, com nível de proficiência $\theta$, escolher a categoria $k$ ou alguma maior que ela no item $i$ é dada por:

$$
P_{i, k}^{+}(\theta)=\frac{1}{1+\exp \left[-a_{i}\left(\theta-b_{i, k}\right)\right]},
$$

sendo $b_{i, k}$ o parâmetro de dificuldade da categoria $k$ do item $i, P_{i, 0}^{+}=1$ e $a_{i}$, como definido anteriormente. Além disso, $b_{i, 0} \leq b_{i, 1} \leq b_{i, 2}$.

A Figura 2.4 ilustra o gráfico da probabilidade acumulada sob o modelo de Samejima com 3 categorias de resposta.

A probabilidade de um individuo, com nível de proficiência $\theta$, escolher a categoria $k$ no item $i$ é:

$$
P_{i, k}(\theta)=P_{i, k}^{+}(\theta)-P_{i, k+1}^{+}(\theta),
$$

com $P_{i, 3}^{+}(\theta)=0$, por definição. Na Figura 2.5 tem-se um exemplo da curva das probabilidades de escolha da categoria sob o modelo de Samejima para 3 categorias de resposta. 


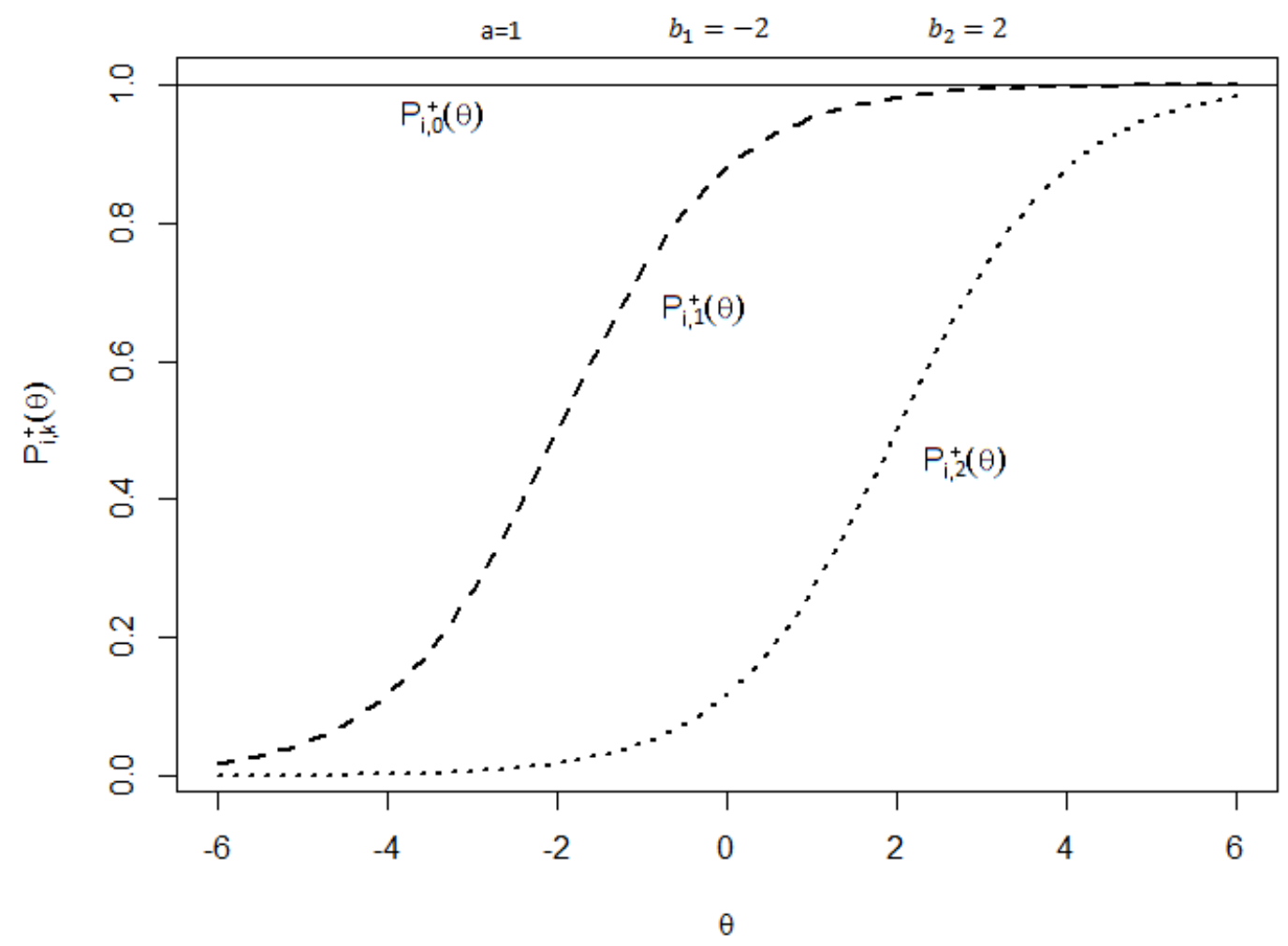

Figura 2.4: Exemplo de uma curva de probabilidade acumulada sob o modelo de Samejima com 3 categorias de resposta.

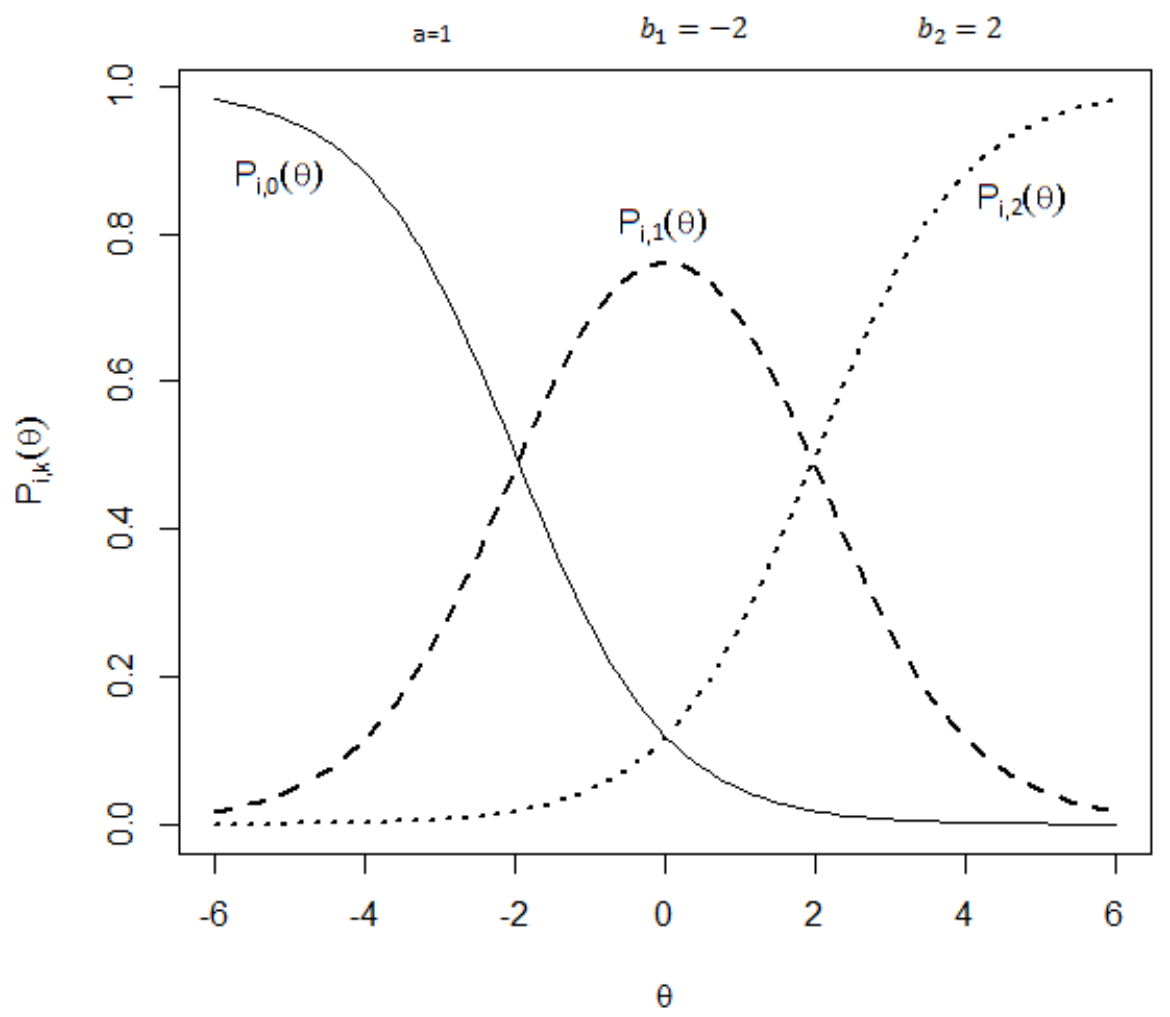

Figura 2.5: Exemplo de uma curva de probabilidade de escolha da categoria sob o modelo de Samejima com 3 categorias de resposta. 
Supondo $l-1$ itens já administrados, a função de verossimilhança do modelo de Samejima é:

$$
L(\theta \mid \boldsymbol{x})=\prod_{i=1}^{l-1} f\left(\boldsymbol{x}_{i} \mid \theta\right)=\prod_{i=1}^{l-1} \prod_{k=0}^{2} P_{i, k}^{x_{i k}}(\theta),
$$

em que $P_{i, k}(\theta)$ é dado em $2.2, k=0,1,2$ representa as categorias dos itens, $\boldsymbol{x}=\left(\boldsymbol{x}_{1}, \ldots, \boldsymbol{x}_{l-1}\right), \boldsymbol{x}_{i}=$ $\left(x_{i 0}, x_{i 1}, x_{i 2}\right)$ e $x_{i k}$ são variáveis que assumem o valor 1 se a categoria $k$ do item $i$ for escolhida e 0 , caso contrário.

\subsection{Estimação do traço latente}

O método EAP foi usado para a estimação de $\theta$, seguindo o conhecido fato da estimação bayesiana funcionar melhor em TAI. Baker (2001),Baker e Kim (2004), Mislevy e Stocking (1989) sugeriam que a estimativa pontual de $\theta$ seja obtida por EAP porque o método pode ser calculado sem a necessidade de métodos iterativos, o que diminiu tempo de estimação. A distribuição, esperança e variância a posteriori de $\theta$, condicionadas aos dados observados, são dadas, respectivamente, pelas expressões 2.4, 2.5 e 2.6 .

$$
\begin{gathered}
\pi(\theta) \propto L(\theta \mid \boldsymbol{x}) g(\theta \mid \boldsymbol{\lambda}), \\
E(\theta \mid \boldsymbol{x}, \lambda)=\frac{\int_{\mathbb{R}} \theta L(\theta \mid \boldsymbol{x}) g(\theta \mid \lambda) d \theta}{\int_{\mathbb{R}} L(\theta \mid \boldsymbol{x}) g(\theta \mid \lambda) d \theta}, \\
\operatorname{Var}(\theta \mid \boldsymbol{x}, \lambda)=\frac{\int_{\mathbb{R}}(\theta-E(\theta))^{2} L(\theta \mid \boldsymbol{x}) g(\theta \mid \boldsymbol{\lambda}) d \theta}{\int_{\mathbb{R}} L(\theta \mid \boldsymbol{x}) g(\theta \mid \boldsymbol{\lambda}) d \theta},
\end{gathered}
$$

em que $g(\theta \mid \boldsymbol{\lambda})$ é a distribuição a priori de $\theta$ e $\boldsymbol{\lambda}$ são os hiperparâmetros.

O método numérico de quadratura gaussiana (Dehghan et al, 2006) foi utilizado para o cálculo das integrais.

\subsection{Seleção de itens}

Para a seleção dos itens a cada passo do TAI-PI, não foi adotado o critério mais tradicional da literatura (informação de Fisher). Isto porque se a estimativa corrente de $\theta$ for distante do seu valor real, este critério pode ser inadequado. Num TAI, especialmente nos passos iniciais, é muito possível que isso ocorra.

Para o i-ésimo item, a informação de Fisher para um nível de traço latente $\theta$ é definida por:

$$
I_{i}(\theta)=E\left[\left(\frac{\partial}{\partial \theta} \log f\left(\boldsymbol{X}_{\boldsymbol{i} . \mid} \mid \theta\right)\right)^{2}\right]
$$

com $f\left(X_{i .} \mid \theta\right)$ a função de probabilidade da resposta dada pelo modelo 2.2 , ou seja, especificamente para o modelo de Samejima. Pode-se observar que 2.7 é calculada em função de $\theta$, que é substituido pela estimativa corrente. Se essa estimativa estiver muito distante do verdadeiro valor, a informação de Fisher será maximizada em um ponto distante do real e, portanto, pode estar muito equivocada.

A informação de Kullback-Leibler, a qual mede a distância entre duas verossimillhanças para um mesmo espaço paramétrico, é que foi escolhida para implementação no TAI-PI, conforme sugerido por Chang e Ying (1996). Quanto maior o valor dessa informação, maior a discrepância entre as duas funções. A medida da informação de Kullback-Leibler para o i-ésimo item no teste entre os níveis de traço latente estimado, $\widehat{\theta}$, e real $\theta$, no modelo de Samejima, é dada por: 


$$
\begin{aligned}
K_{i}(\theta, \widehat{\theta}) & =E\left[\log \frac{f\left(\boldsymbol{X}_{i} \mid \theta\right)}{f\left(\boldsymbol{X}_{i} \mid \widehat{\theta}\right)}\right] \\
& =\sum_{k=0}^{2} P_{i, k}(\theta) \log \frac{P_{i, k}(\theta)}{P_{i, k}(\widehat{\theta})}
\end{aligned}
$$

Vale lembrar que, como temos independência condicional entre as respostas, a informação de Kullback-Leibler após $l-1$ itens administrados é escrita como:

$$
K_{l-1}(\theta, \widehat{\theta})=\sum_{i=1}^{l-1} K_{i}(\theta, \widehat{\theta}) .
$$

Uma vez que o verdadeiro valor do traço latente $(\theta)$ é desconhecido, Sands and Waters (1996) propuseram considerar (2.8) em um intervalo de confiança do traço latente $\left[\widehat{\theta}-\delta_{l}, \widehat{\theta}+\delta_{l}\right]$, sendo $\delta_{l}$ uma função decrescente em relação a 1 (passo corrente do TAI). Assim o critério para selecionar o próximo item a ser apresentado no teste é dado por:

$$
i_{l} \equiv \arg \max _{i}\left\{\int_{\widehat{\theta}-\delta_{l}}^{\widehat{\theta}+\delta_{l}} K_{i}(\theta, \widehat{\theta}) d \theta: i \epsilon L\right\}
$$

sendo $\delta_{l}=z_{\gamma} / \sqrt{l}$ e L, o conjunto dos itens ainda não apresentados ao indivíduo no teste.

\subsection{Shadow test}

O critério de seleção expresso em (2.10) não leva em consideração nenhuma restrição subjetiva para a composição do teste. Por exemplo, uma avaliação adequada da proficiência em inglês deve contemplar itens associados a cada um dos três modulos: estrutura de texto científico, compreensão de texto e convenções gramaticais da língua. A não imposição dessa restrição pode gerar testes com nenhum item de um dos módulos e um número excessivo de itens de outro, distorcendo a avaliação desejada. Outra necessidade de ordem prática diz respeito ao número de itens e de textos no teste. Dois indivíduos que realizam testes com números (e tamanhos) de textos muito distoantes ou com números de itens muito diferentes podem gerar a sensação de injustiça na comparação das estimativas das respectivas proficiências. O indivíduo que respondeu um teste com muitos textos ou muito longo pode reclamar que o cansaço foi maior e, portanto, o desgaste ao longo da realização da prova pode ter influenciado de forma não equiparável entre os indivíduos. Por outro lado, aquele que respondeu um teste curto, com poucos itens, pode reclamar que não houve "tempo"(i.e., número de itens) suficiente para que sua real proficiência seja evidenciada.

Uma das propostas mais interessantes na literatura para uma fácil inclusão de restrições desse tipo é a abordagem Shadow test (Van der Linden 2000). O método de otimização de programação linear inteira é usado para obtenção de um subconjunto (de tamanho $n$, previamente fixado) de itens do banco que maximiza a informação (2.9), tomando $l-1=n$, sujeita às restrições de interesse, também previamente definidas. Um conjunto de $n$ itens é obtido em cada passo do algorítmo, gerando um teste como conjunto solução. É importante destacar que uma das restrições do teste no passo $l$ é que todos os itens já administrados anteriormente estão presentes no teste corrente. $O$ item a ser administrado no passo $l$ do teste é aquele não apresentado nos passos anteriores, que pertença ao conjunto solução do passo corrente e que apresente a máxima informação (2.8) para o atual valor estimado $\hat{\theta}$. Depois do item ser administrado, o restante dos itens não utilizados no teste voltam para o banco de itens como itens disponíveis a serem administrados nas próximas seleções do Shadow test. Uma representação bastante ilustrativa do funcionamento do Shadow test encontra-se na Figura 2.6. 


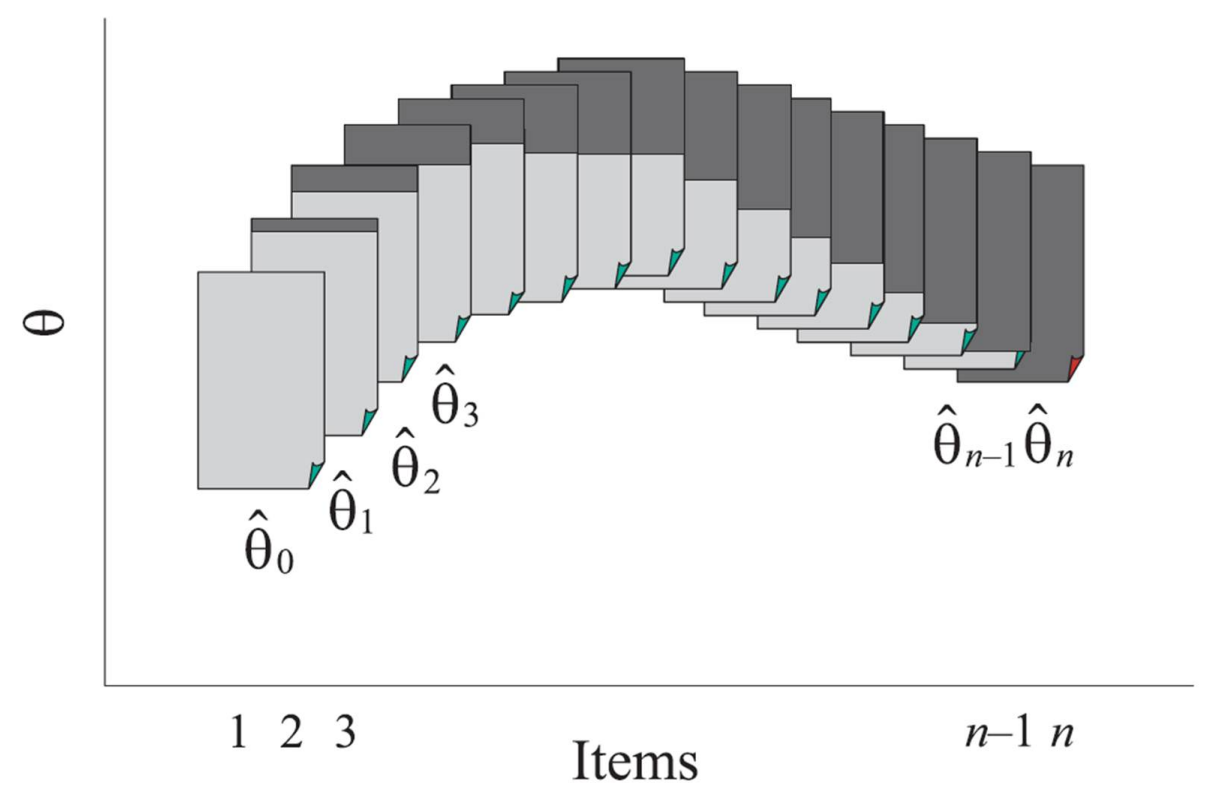

Figura 2.6: Representaçao do Shadow test. Fonte: van der Linden, 2010.

Um especial cuidado deve ser tomado na presença de itens associados a um mesmo texto ou figura (genericamente denominado "estímulo"). Em situações reais, o ideal é apresentar todos os itens associados ao mesmo estímulo sequencialmente (Van der Linden e Glaş 2010; Van der Linden e Pashley, 2010). Neste trabalho, quando um estímulo novo aparece em uma certa iteração do Shadow test, todos os itens associados àquele estímulo no Shadow test dessa iteração irão ser apresentados consecutivamente ao indivíduo.

\subsection{Critério de início e parada}

O nível de conhecimento inicial é a estimativa inicial provisória da proficiência, necessária para o início do teste e relacionada com o nível de dificuldade da primeira questão. Conejo et al. (2001) considera que o nível de dificuldade da primeira questão deve ser escolhido de forma a possibilitar uma redução no tempo de teste. Sukamolson (2002) salienta que usualmente a primeira questão possui um nível de dificuldade médio. Como a princípio não se sabe qual pe a habilidade do respondente, um item médio é a melhor opção inicial.

Como nenhuma informação prévia da proficiência dos alunos é conhecida decidiu-se iniciar com um valor médio $\widehat{\theta}_{0}=0$ para todos.

O critério de parada do teste define o momento em que nenhum item mais é necessário ser respondido pelo indivíduo. Há dois critérios de paradas mais usados: o mais comum por numéro de itens administrados e o outro é até ser atingido uma precisão da estimativa do traço latente, ou seja, um valor pré-determinado do erro padrão. Neste trabalho foi considerado a prova com um número fixo de itens. Isso é exigido pelo Shadow test e é bastante coerente com o EPI, uma vez que a prova tem tempo máximo de 2 horas para ser aplicada e que não se deseja que os alunos respondam a um número muito diferente de itens para evitar questionamentos. 


\section{Capítulo 3}

\section{Estudo de simulação}

Este estudo de simulação foi executado em software $\mathrm{R}$ e delineado para ilustrar o funcionamento de um TAI, em termos da eficiência na estimação do traço latente, comparando distribuições a prioris na utilização do EAP e números diferentes de itens no teste (10, 20, 25 e 30). Para o banco de itens foram simulados 500 itens com 3 categorias de respostas, com $a \sim \operatorname{lognormal}(0.7,0.1)$, gerando apenas bons valores para os parâmetros de dificuldade (Mislevy, 1986), e $b_{i} \sim N(0,1.2)$ com $b_{1}<b_{2}$, com base no modelo de Samejima com 3 categorias de resposta. O método EAP foi utilizado para estimar o traço latente do mesmo modelo e o critério de Kullback-Leibler para a seleção de itens. Como critério de início do TAI foi adotado que todos com $\hat{\theta}_{0}=0$ e como critério de parada número de itens fixos(10,20, 25 e 30 itens).

No primeiro estudo de simulação, para cada $\theta \in\{-3,-2,-1,0,1,2,3\}$ foram simulados 200 testes. Adicionalmente para cada teste simulado, três tipos de distribuições a priori para as habilidades foram adotadas para o método EAP: $\mathrm{N}(0,1)$, que é usualmente adotada na literatura, $\mathrm{N}(0,2)$, para dar menos informação sobre o parâmetro em questão e $N\left(\hat{\theta}_{i-1}, \sigma\left(\hat{\theta}_{i-1}\right)\right)$ para aproveitar a estimativa corrente como informação a priori da habilidade do indivíduo. Totalizando assim $7 x 3 x 200=4200$ testes simulados.

Para avaliar a qualidade do modelo, foram calculadas as medidas a seguir:

$$
\begin{gathered}
\text { Vicio }=\sum_{j=1}^{200} \frac{\hat{\theta}_{j l}-\theta_{l}}{100}, \\
E Q M=\frac{\sum_{j=1}^{200}\left(\hat{\theta}_{j l}-\theta_{l}\right)^{2}}{100},
\end{gathered}
$$

em que $j=1, \ldots, 200$ representa as repetições de cada valor fixado de $\theta, l=1, \ldots, 7$ indexa os 7 valores fixados para $\theta, \theta_{l} \in\{-3,-1,-2,0,1,2,3\}$ são os valores fixados para $\theta, \hat{\theta}_{j l}$ é a estimativa de $\theta_{l}$ obtida pelo método EAP na simulação. Os valores encontrados são apresentados nas Tabelas 3.1 e 3.2, nas quais pode-se observar que o vício se aproxima de zero e o erro quadrático médio diminui conforme aumenta o número de itens e que o ganho em precisão de estimação aumentando o tamanho do teste de 25 e 30 talvez não seja importante e 20 itens já são suficientes em um teste. Com exceção da priori $N\left(\hat{\theta}_{i-1}, \sigma\left(\hat{\theta}_{i-1}\right)\right)$, as estimativas foram muito próximas dos valores reais para as demais prioris apresentadas, o que pode ser confirmado pelos baixos valores do vício e do erro quadrático médio, no entando aumentam consideralvelmente quando se é usado $\mathrm{N}(0,1)$ como priori para valores de habilidades muito distantes da média (ou seja, iguais a $-3,3$ ). 
Tabela 3.1: Vício dos estimadores para diferentes prioris.

\begin{tabular}{c|c|ccccccc}
\hline \hline Priori & Itens no & \multicolumn{7}{|c}{ Valores reais de $\theta$} \\
\cline { 3 - 9 } & teste & -3 & -2 & -1 & 0 & 1 & 2 & 3 \\
\hline \multirow{3}{*}{$\mathrm{N}(0,1)$} & 10 & 0.3978 & 0.1478 & 0.0636 & 0.0057 & -0.0707 & -0.1050 & -0.6543 \\
& 20 & 0.1756 & 0.0768 & 0.0373 & 0.0180 & -0.0423 & -0.0246 & -0.5341 \\
& 25 & 0.1403 & 0.0451 & 0.0175 & 0.0104 & -0.0256 & -0.0112 & -0.5029 \\
& 30 & 0.1200 & 0.0497 & 0.0168 & 0.0045 & -0.0154 & -0.0093 & -0.4754 \\
\hline \multirow{3}{*}{$\mathrm{N}(0,2)$} & 10 & 0.1020 & 0.0569 & -0.0270 & -0.0149 & -0.0199 & 0.1536 & -0.1768 \\
& 20 & 0.0467 & 0.0222 & -0.0144 & -0.0110 & -0.0032 & 0.1192 & -0.0814 \\
& 25 & 0.0482 & 0.0237 & -0.0086 & -0.0134 & -0.0043 & 0.1064 & -0.0636 \\
& 30 & 0.0486 & 0.0241 & -0.0100 & -0.0099 & -0.0037 & 0.1153 & -0.0558 \\
\hline \multirow{3}{*}{$N\left(\hat{\theta}_{i-1}, \sigma\left(\hat{\theta}_{i-1}\right)\right)$} & 10 & 0.5851 & 0.2588 & 0.0557 & -0.0056 & -0.0599 & -0.1677 & -0.7912 \\
& 20 & 0.3225 & 0.1229 & 0.0404 & -0.0010 & -0.0311 & -0.0718 & -0.6824 \\
& 25 & 0.2759 & 0.0971 & 0.0228 & -0.0038 & -0.0337 & -0.0550 & -0.6565 \\
& 30 & 0.2391 & 0.0820 & 0.0210 & -0.0014 & -0.0287 & -0.0536 & -0.6352 \\
\hline \hline
\end{tabular}

Tabela 3.2: Erro Quadrático Médio dos estimadores para diferentes prioris.

\begin{tabular}{c|c|ccccccc}
\hline \hline \multirow{2}{*}{ Priori } & Itens no & \multicolumn{7}{|c}{ Valores reais de $\theta$} \\
\cline { 3 - 9 } & teste & -3 & -2 & -1 & 0 & 1 & 2 & 3 \\
\hline \multirow{3}{*}{$\mathrm{N}(0,1)$} & 10 & 0.2498 & 0.1295 & 0.0760 & 0.0735 & 0.0790 & 0.1015 & 0.4478 \\
& 20 & 0.0795 & 0.0525 & 0.0430 & 0.0395 & 0.0427 & 0.0662 & 0.3105 \\
& 25 & 0.0568 & 0.0397 & 0.0355 & 0.0314 & 0.0352 & 0.0663 & 0.2800 \\
& 30 & 0.0463 & 0.0397 & 0.0325 & 0.0249 & 0.0304 & 0.0608 & 0.2559 \\
\hline \multirow{3}{*}{$\mathrm{N}(0,2)$} & 10 & 0.0899 & 0.1164 & 0.0896 & 0.0778 & 0.0962 & 0.2381 & 0.0923 \\
& 20 & 0.0516 & 0.0580 & 0.0550 & 0.0438 & 0.0400 & 0.1551 & 0.0675 \\
& 25 & 0.0426 & 0.0462 & 0.0492 & 0.0339 & 0.0321 & 0.1350 & 0.0667 \\
& 30 & 0.0371 & 0.0394 & 0.0395 & 0.0287 & 0.0308 & 0.1391 & 0.0730 \\
\hline \multirow{3}{*}{$N\left(\hat{\theta}_{i-1}, \sigma\left(\hat{\theta}_{i-1}\right)\right)$} & 10 & 0.4508 & 0.1217 & 0.0872 & 0.1041 & 0.0764 & 0.0920 & 0.6352 \\
& 20 & 0.1641 & 0.0539 & 0.0433 & 0.0487 & 0.0416 & 0.0502 & 0.4755 \\
& 25 & 0.1251 & 0.0446 & 0.0392 & 0.0358 & 0.0356 & 0.0474 & 0.4408 \\
& 30 & 0.0953 & 0.0427 & 0.0339 & 0.0305 & 0.0280 & 0.0473 & 0.4133 \\
\hline \hline
\end{tabular}

Contemplando apenas os tamanhos de teste de 20, 25 e 30 itens e aumentando o número de habilidade geradas de 200 para 1000, realizou-se uma segunda simulação com o intuito de constrastar graficamente os valores estimados e verdadeiros. Nas Figuras 3.1 e 3.2 são apresentadas a proficiência verdadeira em função da estimada e os obtidos respectivamente, pode-se observar que para todas as distribuições a prioris consideradas e número de itens no teste as estimações são bem próxima dos valores reais. Este fato auxiliará na escolha do número de itens a serem apresentados na prova. 

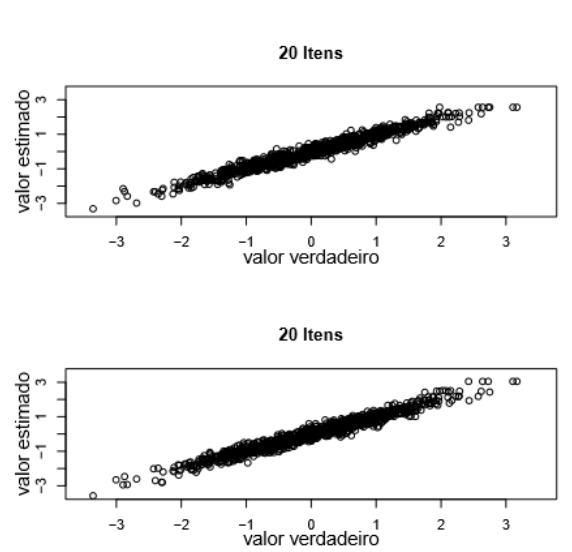

20 Itens

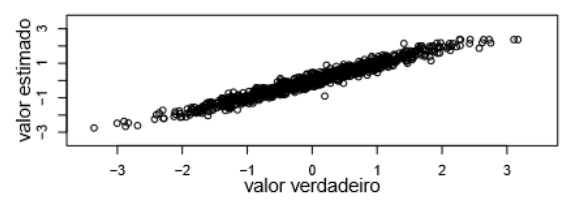

$\mathrm{N}(0,1)$

25 Itens

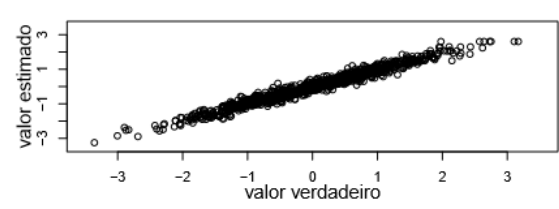

$\mathrm{N}(0,2)$

25 Itens

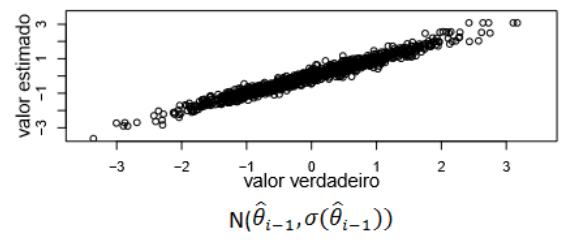

25 Itens

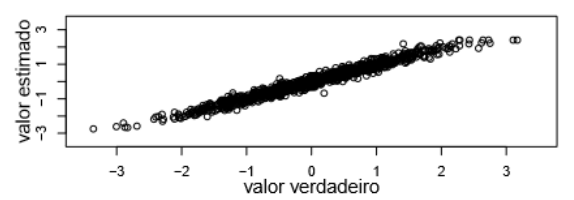

30 Itens

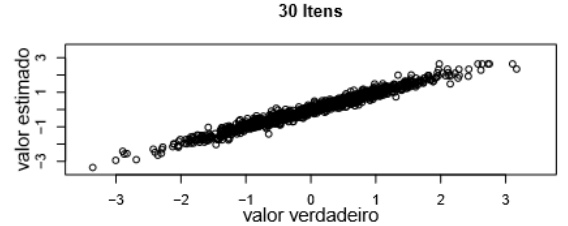

30 Itens

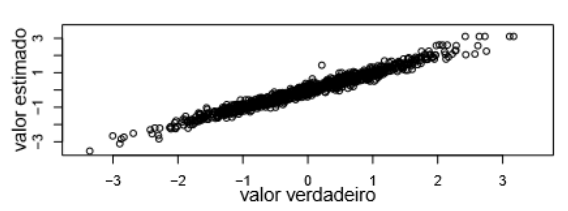

30 Itens

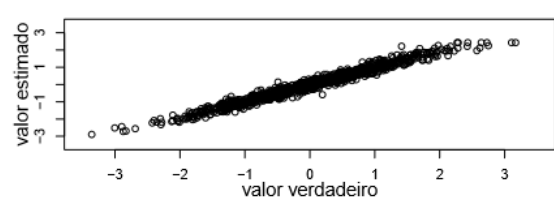

Figura 3.1: Valor verdadeiro em função do valor estimado de $\theta$ por 3 prioris, para diferentes tamanhos de teste (20, 25 e 30 itens)
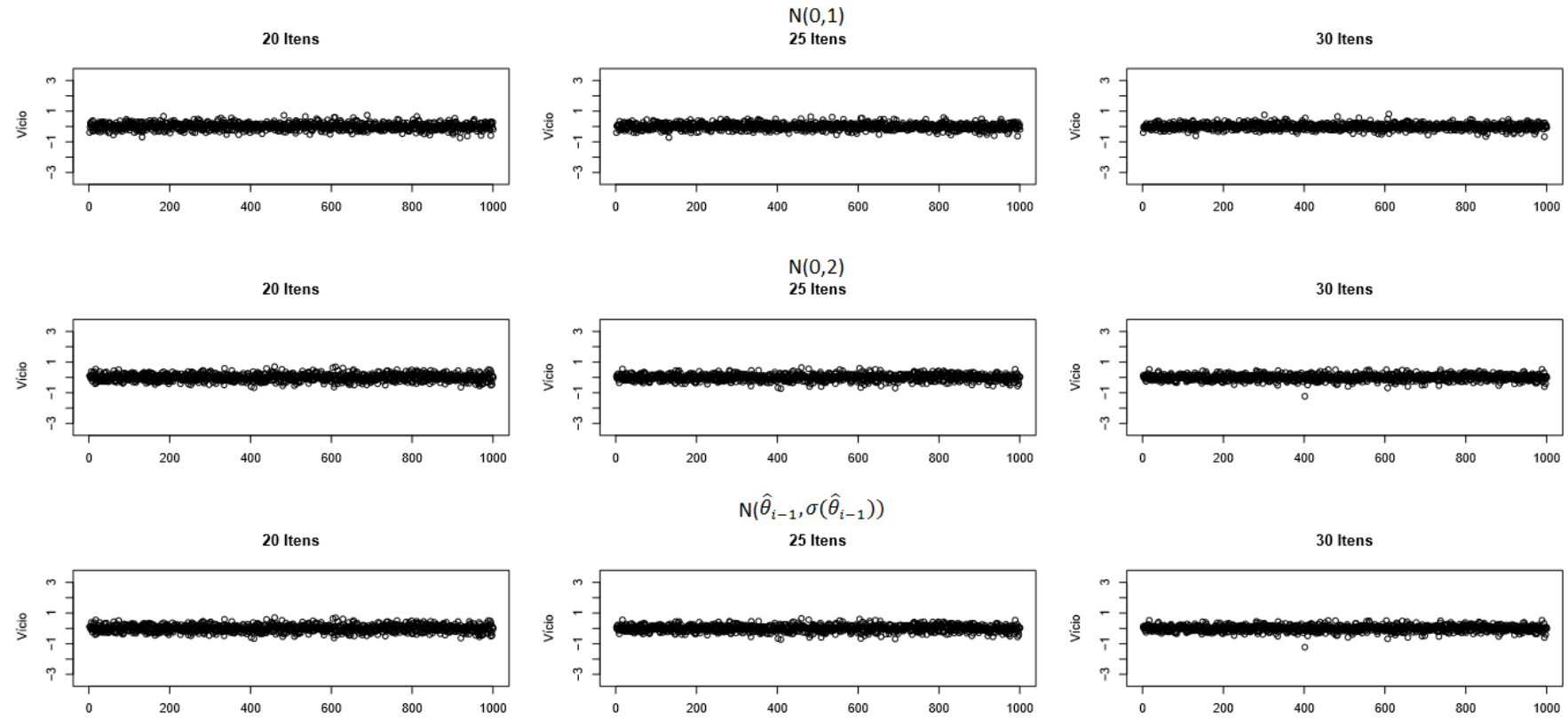

Figura 3.2: Vício de 1000 respondentes simulados estimado por 3 prioris, para diferentes tamanhos de teste(20, 25 e 30 itens) 


\section{Capítulo 4}

\section{Aplicação real}

As aplicações reais do TAI-PI foram baseadas a partir dos 167 itens criados e aplicados no periodo de 2002 a 2012 no EPI formato lápis-e-papel. No entanto para as aplicações foram filtrados 74 itens para a calibração dos parâmetros, o quais tinham ao menos 80 respondentes ao longo do tempo (para obterem-se estimativas relativamente confiáveis dos parâmetros)e presentes em provas comuns (para garantir a equalização simultânea com a estimação dos parâmetros do modelo da TRI). Desses itens foram selecionados os que tinham parâmetros de discriminação (a) acima de 0,7, associados a nenhum texto ou com pelo menos 3 itens associados a um texto. As estimativas dos parâmetros dos itens que compõem esse banco foram obtidas na métrica(0,1) (de Andrade et al. , 2000) utilizando-se as respostas resultantes das aplicações desde 2002. Sobrando asssim depois de várias filtragem 40 itens para o banco que foram utilizados em aplicações reais e também usados para o estudo do número de categorias que deveriam ser consideradas no modelo de Samejima.

A primeira aplicação do TAI-PI ocorreu em novembro de 2013 a 26 alunos de pós-graduação do CCMC. Como o método de avaliação proposto no TAI-PI ainda não havia sido validado e não se sabia nem mesmo um ponto de corte adequado na escala do traço latente para aprovação ou reprovação do aluno, optou-se por usar a ocasião apenas para um teste do funcionamento do software. Na realidade, os alunos responderam todos os mesmo 25 itens (pré-escolhidos dentre todos os 40 calibrados do banco), com variação na ordem de apresentação segundo a maximização da informação de Kullback-Leibler, conforme explicado anteriormente. A aplicação indentificou um problema de autorização para salvamento das respostas em alguns computadores devido à forma incorreta de efetuar o login na rede da sala por alguns alunos. O problema foi solucionado e inserindo uma verificação de autorização de acesso já no início do teste, nas versões subsequentes do TAI-PI, e emitindo um aviso se o acesso for negado.

A segunda aplicação do TAI-PI foi realizada em maio de 2014 por 59 alunos da pós graduação do CCMC-ICMC e do Programa Interinstitucional de Pós Graduação em Estatística (PIPGES). Foram selecionados para compor o banco 40 itens, com as mesmas restrições de parâmetro de discriminação, associadas a texto citadas acima na primeira aplicação. Nessa fase queria-se testar o funcionamento do TAI-PI em uma prova real composta de 25 itens selecionados de um banco de 40 itens, pelo método explicado no Capítulo 2 e obedecendo a algumas restrições de conteúdo. Todos os alunos responderam os 40 itens (pois o critério de correção ainda seria pelo MPA), sendo que apenas os 25 primeiros itens foram selecionados de forma adaptativa (conforme metodologia no Capítulo 3 e os demais 15 itens foram apresentados sequencialmente. A estrutura dos 40 itens é apresentada na Tabela 4.1 
Tabela 4.1: Banco de itens do TAI-PI, maio de 2014

\begin{tabular}{c|c|c}
\hline Módulo & Estímulo & Número de Itens \\
\hline \multirow{3}{*}{1} & Abstract 1 & 3 \\
& Abstract 2 & 4 \\
& Abstract 3 & 3 \\
& Abstract 4 & 4 \\
\hline \multirow{2}{*}{2} & Introduction 5 & 7 \\
& Introduction 6 & 4 \\
\hline 3 & Nenhum & 15 \\
\hline
\end{tabular}

Para a seleção dos 25 primeiros itens de forma adaptativa, foram impostas as seguintes restrições de conteúdo e tamanho de textos, implementados via Shadow test (Van der Linden 2000):

- Exatamente 3 textos do módulo 1 (com no mínimo 3 e no máximo 4 itens cada texto)

- Exatamente 1 texto do módulo 2 (com no mínimo 4 e no máximo 7 itens)

- Nó máximo 11 itens do módulo 3.

- No máximo 2015 palavras contidas nos textos contemplando pelos 25 itens.

A prova foi realizada sem maiores intercorrências. Os dados obtidos correspondem, além das respostas a todos os itens do banco, ao traço latente estimado e respectivo erro padrão em cada um dos 25 passos do TAI e, também, após os 40 itens do banco serem respondidos. A Tabela 4.2 traz as estimativas do traço latente dos alunos após o teste adaptativo de 25 itens e após a resposta a todos os 40 itens do banco, assim como a classificação (em aprovado ou reprovado) com base no MPA aplicado às 40 respostas. Como pode-se observar destacado em cinza, 4 alunos estão com estimativas baixas e foram aprovados. Note, pela Tabela 4.3, que os resultados desses indivíduos correspondem ao mínimo necessário (ou quase isso, no caso do aluno 17) para aprovação pelo MPA. Pode-se interpretar tal fato como uma amenização dessa contradição entre os métodos (MPA e TAI) 
Tabela 4.2: Estimativas dos traços latentes (após 25 e 40 itens respondidos) e classificação pelo MPA na aplicaçao do TAI- de maio de 2014.

\begin{tabular}{|c|c|c|c|c|}
\hline Número & Indivíduo & $\widehat{\theta}_{25}$ & $\widehat{\theta}_{40}$ & MPA \\
\hline 1 & 10 & $-2,07$ & $-1,68$ & REFAZER \\
\hline 2 & 1 & $-1,62$ & $-1,66$ & REFAZER \\
\hline 3 & 6 & $-1,29$ & $-1,60$ & REFAZER \\
\hline 4 & 35 & $-1,39$ & $-1,59$ & REFAZER \\
\hline 5 & 37 & $-1,-19$ & $-1,44$ & REFAZER \\
\hline 6 & 26 & $-1,34$ & $-1,41$ & APROVADO \\
\hline 7 & 52 & $-1,09$ & $-1,37$ & REFAZER \\
\hline 8 & 23 & $-1,37$ & $-1,28$ & REFAZER \\
\hline 9 & 29 & $-0,90$ & $-1,16$ & REFAZER \\
\hline 10 & 9 & $-1,06$ & $-1,15$ & REFAZER \\
\hline 11 & 20 & $-1,07$ & $-1,15$ & REFAZER \\
\hline 12 & 12 & $-0,96$ & $-1,11$ & REFAZER \\
\hline 13 & 38 & $-0,92$ & $-1,08$ & APROVADO \\
\hline 14 & 27 & $-1,01$ & $-0,99$ & REFAZER \\
\hline 15 & 3 & $-0,28$ & $-0,76$ & REFAZER \\
\hline 16 & 54 & $-0,29$ & $-0,75$ & REFAZER \\
\hline 17 & 22 & $-0,58$ & $-0,73$ & REFAZER \\
\hline 18 & 47 & $-0,34$ & $-0,69$ & APROVADO \\
\hline 19 & 49 & $-0,55$ & $-0,69$ & REFAZER \\
\hline 20 & 18 & $-0,46$ & $-0,68$ & REFAZER \\
\hline 21 & 28 & $-0,81$ & $-0,66$ & REFAZER \\
\hline 22 & 51 & $-0,62$ & $-0,65$ & REFAZER \\
\hline 23 & 25 & $-0,52$ & $-0,65$ & REFAZER \\
\hline 24 & 2 & $-0,61$ & $-0,64$ & REFAZER \\
\hline 25 & 39 & $-0,71$ & $-0,60$ & REFAZER \\
\hline 26 & 5 & $-0,62$ & $-0,56$ & REFAZER \\
\hline 27 & 11 & $-0,29$ & $-0,54$ & REFAZER \\
\hline 28 & 19 & $-0,17$ & $-0,52$ & REFAZER \\
\hline 29 & 17 & $-0,56$ & $-0,48$ & APROVADO \\
\hline 30 & 40 & $-0,28$ & $-0,41$ & REFAZER \\
\hline 31 & 53 & $-0,58$ & $-0,41$ & REFAZER \\
\hline 32 & 44 & $-0,32$ & $-0,40$ & APROVADO \\
\hline 33 & 42 & $-0,01$ & $-0,34$ & APROVADO \\
\hline 34 & 45 & $-0,29$ & $-0,24$ & APROVADO \\
\hline 35 & 48 & $-0,03$ & $-0,22$ & APROVADO \\
\hline 36 & 41 & $-0,36$ & $-0,15$ & APROVADO \\
\hline 37 & 31 & $-0,07$ & $-0,15$ & APROVADO \\
\hline 38 & 34 & $-0,22$ & $-0,14$ & APROVADO \\
\hline 39 & 57 & $-0,29$ & $-0,12$ & APROVADO \\
\hline 40 & 50 & 0,19 & $-0,10$ & APROVADO \\
\hline 41 & 58 & 0,00 & $-0,09$ & APROVADO \\
\hline 42 & 33 & $-0,23$ & $-0,04$ & APROVADO \\
\hline 43 & 43 & $-0,01$ & 0,08 & APROVADO \\
\hline 44 & 13 & 0,44 & 0,09 & APROVADO \\
\hline 45 & 55 & 0,11 & 0,13 & APROVADO \\
\hline 46 & 32 & 0,11 & 0,27 & APROVADO \\
\hline 47 & 30 & 0,46 & 0,35 & APROVADO \\
\hline 48 & 15 & 0,21 & 0,38 & APROVADO \\
\hline 49 & 4 & 0,70 & 0,45 & APROVADO \\
\hline 50 & 14 & 0,51 & 0,47 & APROVADO \\
\hline 51 & 16 & 0,49 & 0,51 & APROVADO \\
\hline 52 & 56 & 0,30 & 0,53 & APROVADO \\
\hline 53 & 8 & 0,62 & 0,58 & APROVADO \\
\hline 54 & 21 & 0,42 & 0,67 & APROVADO \\
\hline 55 & 36 & 0,59 & 0,70 & APROVADO \\
\hline 56 & 24 & 0,72 & 0,93 & APROVADO \\
\hline 57 & 7 & 0,89 & 0,94 & APROVADO \\
\hline 58 & 59 & 0,85 & 0,95 & APROVADO \\
\hline 59 & 46 & 1,17 & 1,08 & APROVADO \\
\hline
\end{tabular}


Tabela 4.3: Porcentagem de acertos de 4 alunos em cada categoria na aplicação de maio de 2014.

\begin{tabular}{c|c|c|c|c}
\hline Aluno & \% Total.Informado & \%Informado & \% Parc. Informado & \% Total. Mal Informado \\
\hline 26 & $0 \%$ & $0 \%$ & $90 \%$ & $10 \%$ \\
38 & $50 \%$ & $0 \%$ & $0 \%$ & $25 \%$ \\
47 & $50 \%$ & $12,5 \%$ & $5 \%$ & $25 \%$ \\
17 & $52,5 \%$ & $5 \%$ & $7,5 \%$ & $25 \%$ \\
\hline
\end{tabular}

A Figura 4.1 traz o boxplot das proficiências estimadas no TAI-PI para os 59 alunos classificados em reprovados ou aprovados, segundo o MPA, pode-se verificar um possível valor de corte para a classificação do aluno na escala traço latente em torno de -0.5 e que as proficiências parecem ser bem maiores no grupo aprovado do que no reprovado, com pouca sobreposição entre as duas distribuições do traço latente, mais presente na estimação dos 25 itens do que com 40.
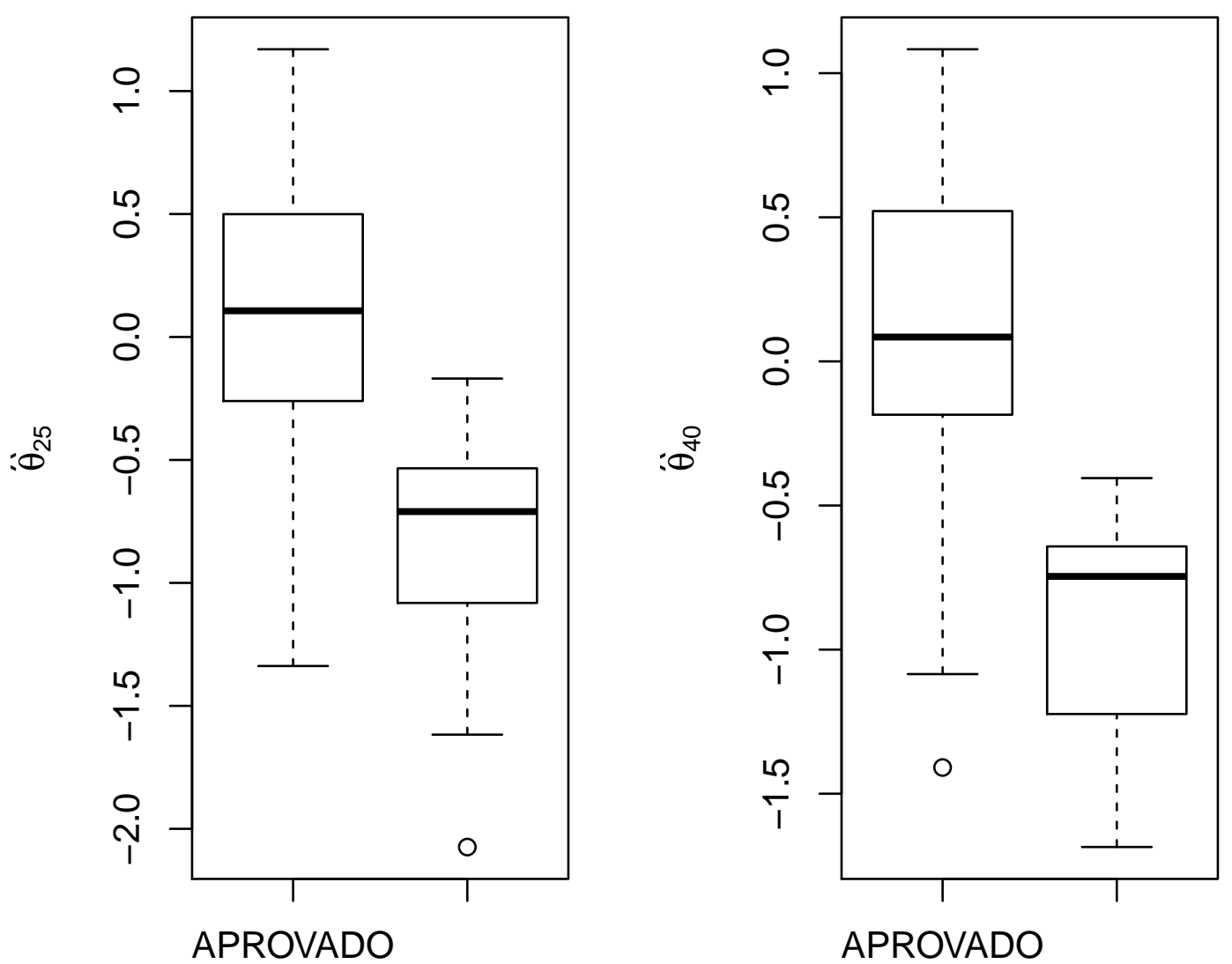

Figura 4.1: Boxplot da proficiência estimada após 25 e 40 itens respondidos dos 59 alunos na aplicação do TAI-PI, separadamente para o grupo reprovado e aprovado pelo critério MPA.

Nas Figuras 4.2, 4.3 e 4.4 são apresentadas proficiências estimadas e seus desvios padrão de alguns alunos (dois deles, com respostas descritas na Tabela 4.3 em cada passo do teste adaptativo, pode-se observar que quando o aluno erra o item (representado pela categoria 0), a proficiência subsequente é estimada num valor menor. Enquanto que, quando o aluno acerta (categoria 2), a proficiência estimada aumenta. 
Além disso, em testes adaptativos informatizados classificatórios encontrados na literatura é comum usar um intervalo de credibilidade para o traço latente no último passo para classificar o aluno como aprovado (se o intervalo todo estiver acima do corte) ou reprovado (se o intervalo estiver abaixo do corte). No caso em que o intercalo contiver o corte, seguir com o teste para ter-se uma estimativa mais precisa de $\widehat{\theta}$. Por esse critério, tanto o aluno 26 , quanto 46, provavelmente estariam classificados após 25 itens respondidos (se considerar-se intervalos com amplitudes de 4 vezes o erro padrão, aproximadamente).

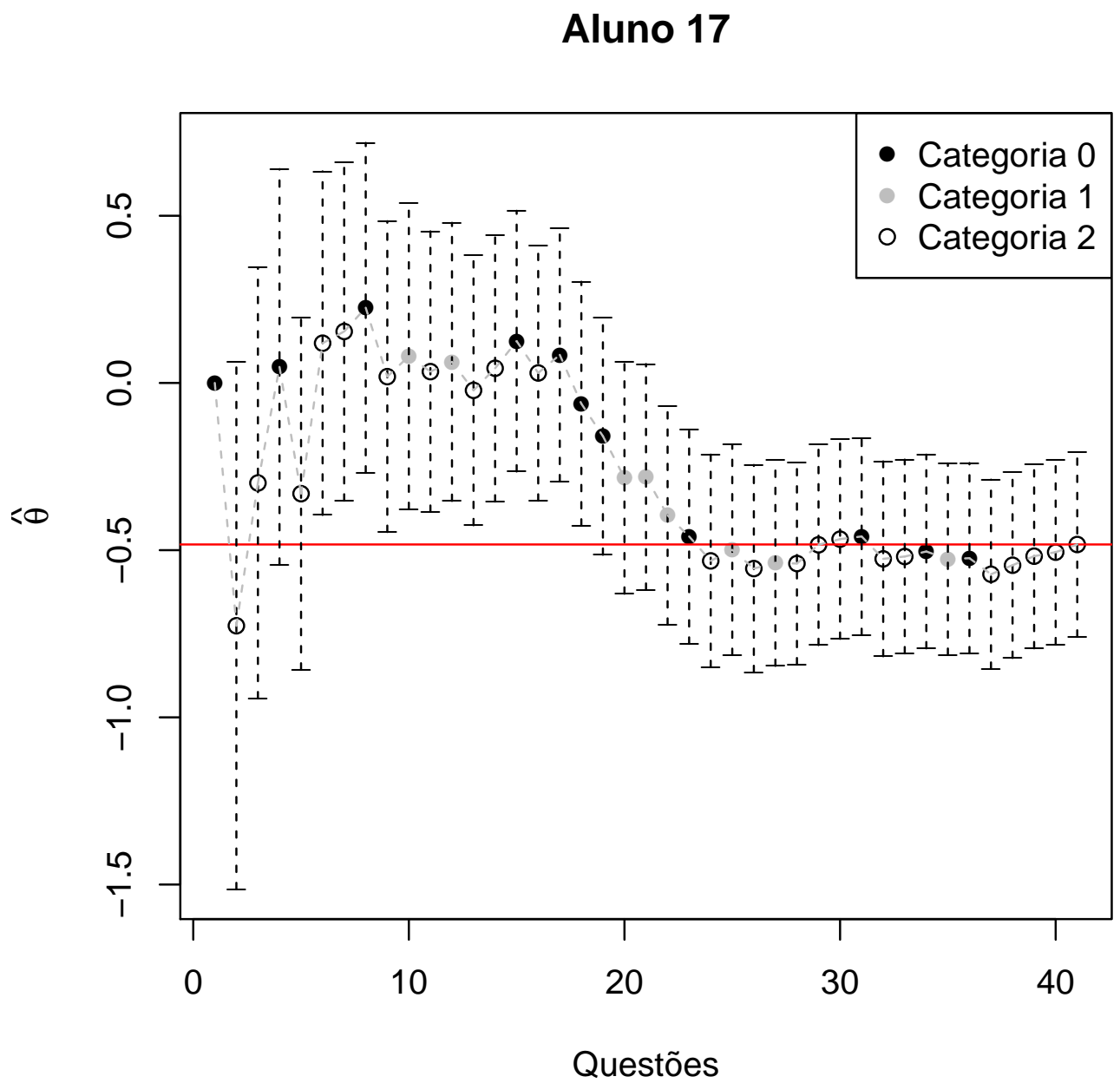

Figura 4.2: Proficiências estimadas do aluno 17 em cada passo do TAI-PI, a linha vermelha representa a estimativa final após 40 itens. 


\section{Aluno 26}

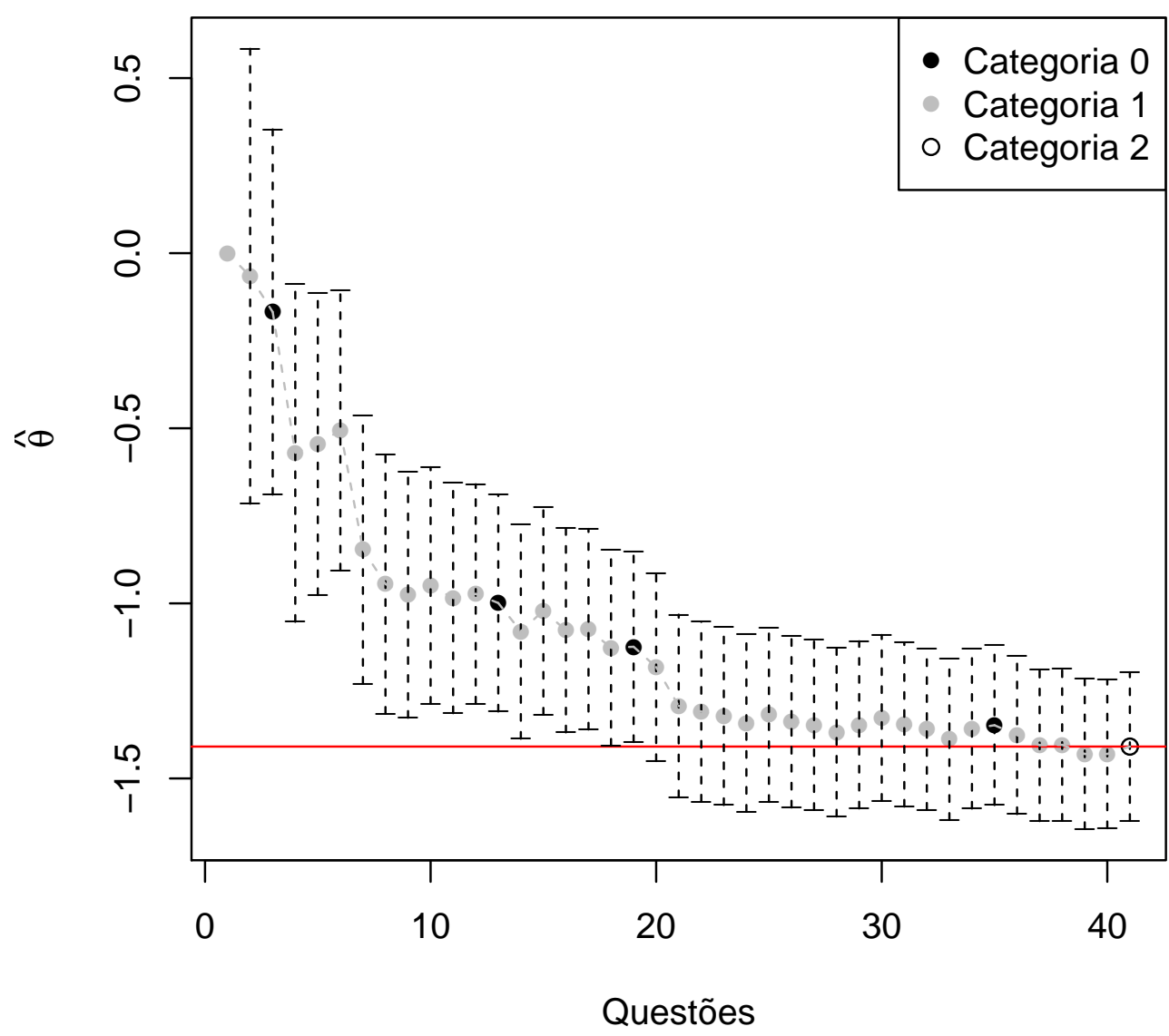

Figura 4.3: Proficiências estimadas do aluno 26 em cada passo do TAI-PI, a linha vermelha representa a estimativa final após 40 itens. 


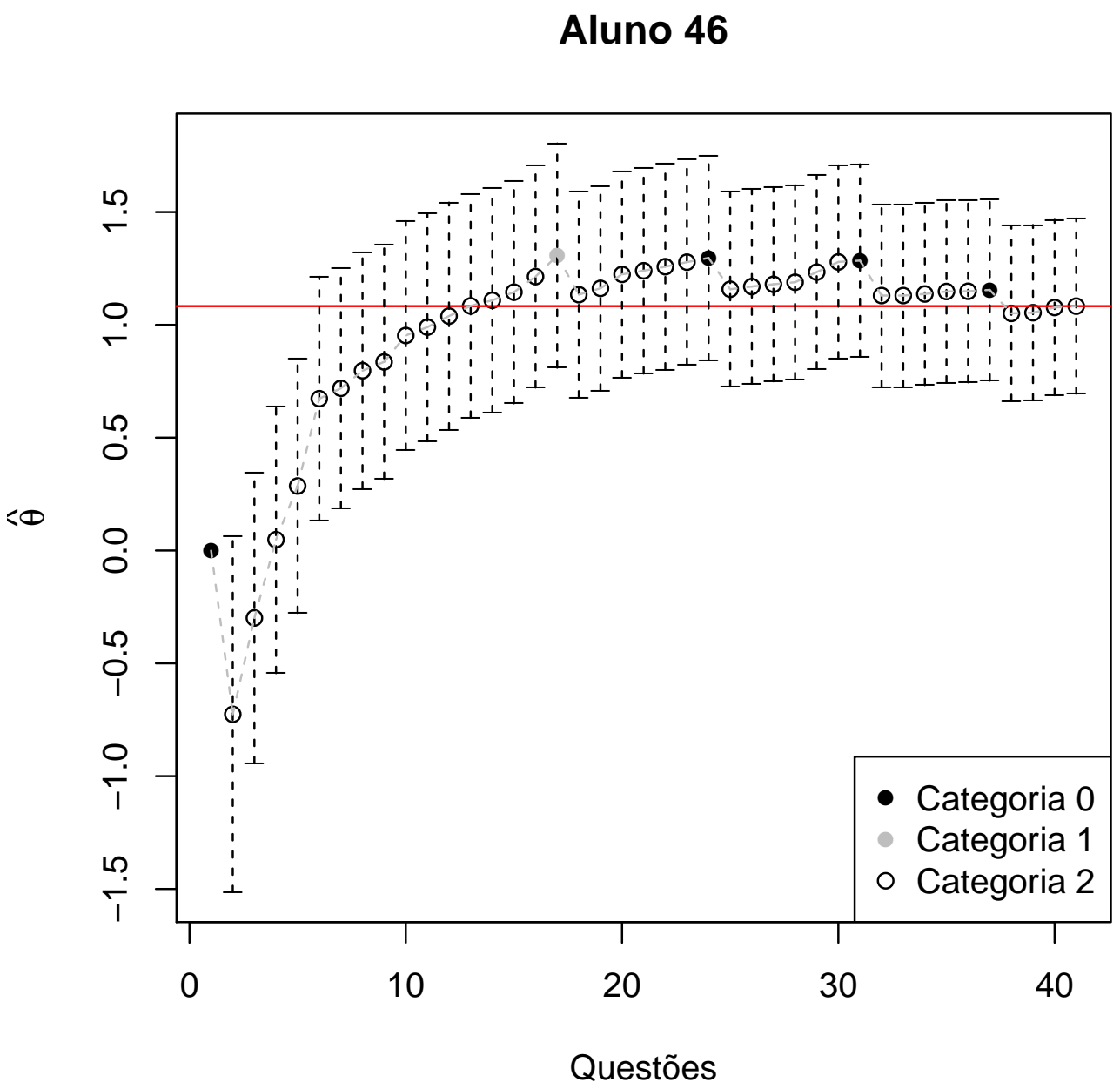

Figura 4.4: Proficiências estimadas do aluno 46 em cada passo do TAI-PI, a linha vermelha representa a estimativa final após 40 itens. 


\section{Capítulo 5}

\section{Estudos de classificação}

O objetivo do EPI não é estimar a proficiência dos alunos, mas classificá-los em aprovados ou reprovados. Assim, é preciso que encontrar um ponto de corte, no qual os alunos com proficiências estimadas acima deste ponto sejam classificados em aprovados e abaixo em reprovados. Para isso foram realizados estudos nos resultados da aplicação no Capítulo 4. Pelo fato de quer-se manter a história e estrutura do EPI, o ponto de corte será baseado no $\widehat{\theta}_{40}$. Mas para medir qualidade e eficiência deste ponto os estudos serão realizados no $\widehat{\theta}_{25}$.

Na Figura 5.1 é apresentado a curva ROC para o $\hat{\theta}_{40}$ baseado no critério MPA, a qual é muito utilizada na área da saúde para medir e especificar problemas no desempenho de testes diagnósticos. Neste trabalho a curva ROC será utilizada para obter o ponto de corte da proficiência.

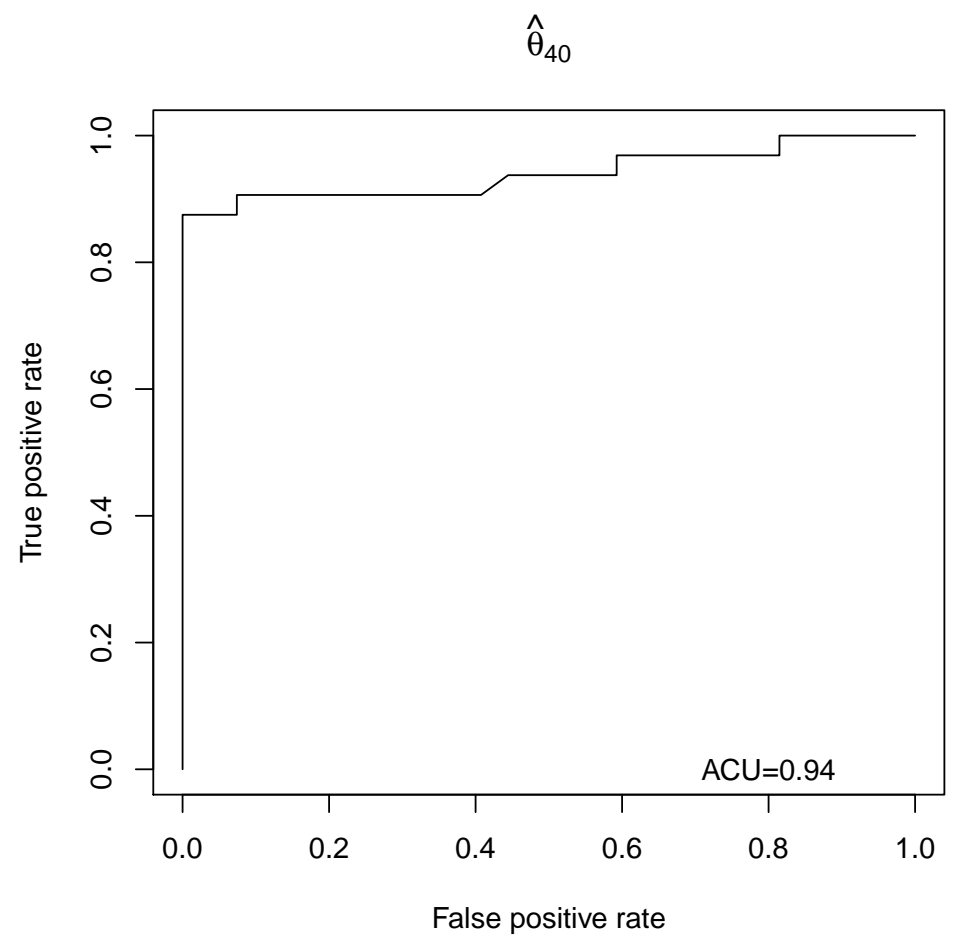

Figura 5.1: Curva ROC para a proficiência de acordo com a classificação MPA

O método custo-benefício (McNeil et al. (1975),Metz (1978)) foi utilizado para encontrar o ponto de corte ótimo, o qual é obtido quando a inclinação da curva ROC é dada por

$$
S=\frac{1-p}{p} C R=\frac{1-p}{p} \frac{C_{F P}-C_{T N}}{C_{F N}-C_{T P}}
$$

onde $p$ é a prevalência de reprovados no teste, $C_{F P}$ o custo do falso positivo, $C_{T N}$ custo do verdadeiro 
negativo, $C_{F N}$ custo do falso negativo e $C_{T P}$ custo do verdadeiro positivo, foi utilizado $C R=1$, o valor encontrado para o $\widehat{\theta}_{40}$ é -0.40 . Na Figura 5.2 são apresentadas as densidades estimadas para a proficiência separadas em aprovados e reprovados, na qual é possível observar pelas caudas sobrepostas que há uma pequena probabilidade de se cometer erros na classificação.

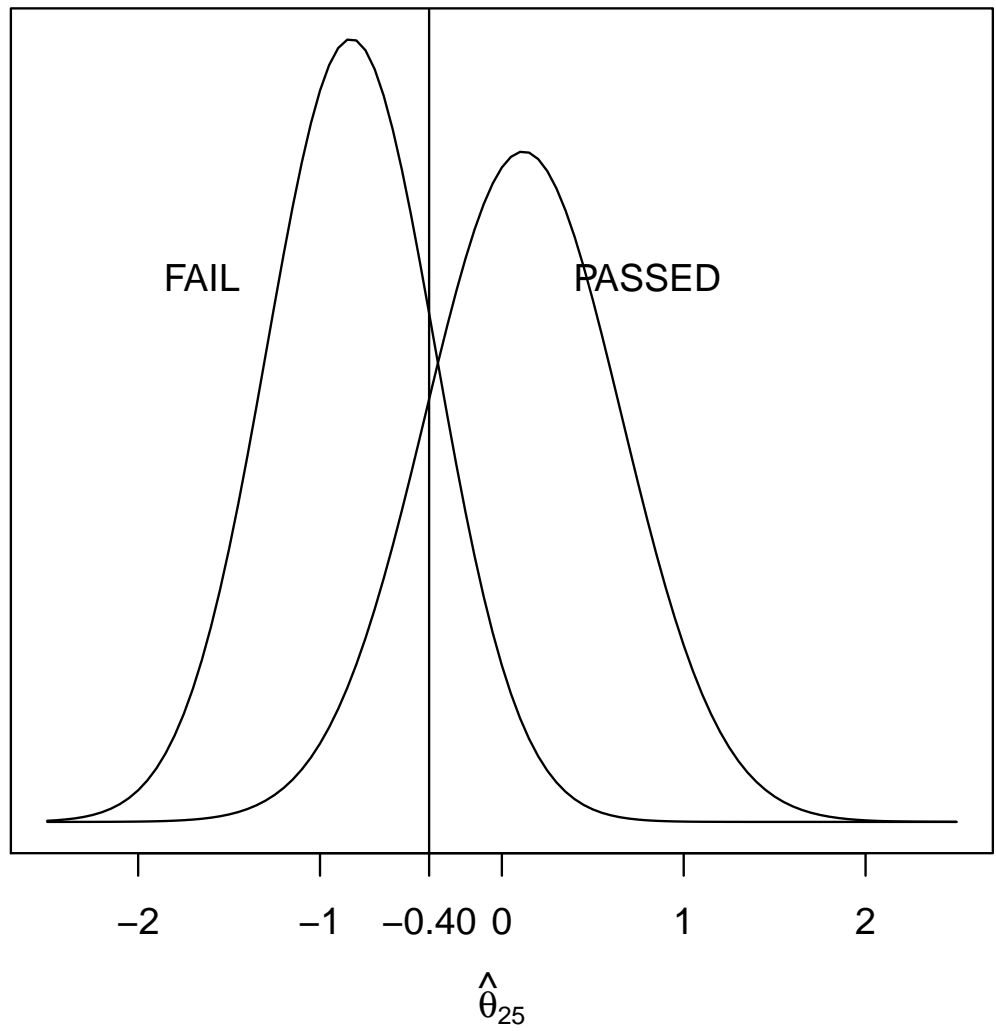

Figura 5.2: Densidades estimadas para a proficiência por grupo

Esse erro de classificação pode ser medido por meio do método Acurácia Esperada da Classificação proposto por Rudner (2005). A acurácia mede o quanto o teste consegue obter resultados verdadeiros, ou seja, no nosso caso quando o aluno classificado em aprovado pelo ponto de corte e aprovado pelo critério MPA ou o caso oposto, reprovado pelo ponto corte e reprovado pelo critério MPA. A acurácia esperada é dada por

$$
A E=\frac{\sum_{\theta_{i}<\theta_{c}} P\left(\hat{\theta}<\theta_{c} \mid \theta_{i}\right)}{n}+\frac{\sum_{\theta_{i}>\theta_{c}} P\left(\hat{\theta}>\theta_{c} \mid \theta_{i}\right)}{n}
$$

onde $\theta_{c}=-0.40$. A acurácia esperada encontrada foi de 0,85 , ou seja, espera que se cometa um erro de $15 \%$ na hora de classificar um aluno, um valor razoávelmente pequeno pelo fato que geralmente o aluno tem uma segunda chance de realizar a prova.

Um estudo de simulação também foi realizado para verificar a eficácia de estimação da proficiência do aluno próximo ao ponto de corte a partir das simulações apresentadas na Seçao 3 e adotando a distribuição a priori para a proficiência do indivídio $\mathrm{N}(0,1)$, ou seja, os mesmo métodos utilizados no TAI-PI. Foram simulados testes de 1000 alunos com proficiências adotadas a partir de uma sequência de -1,4 a 0,6 com espaçamento de 0.002, e foi calculada a acúrácia apresentadas na Tabela 5.1. Obteve-se um erro de classificação de aproximadamente $6 \%$, um número pequeno e geralmente aceitável, o que pode evidenciar a validade do ponto de corte adotado e dos métodos 
utlizados próximo a este ponto.

Tabela 5.1: Acurácia para o ponto de corte no estudo de simualção

\begin{tabular}{c|cc}
\hline & $\theta<-0.4$ & $\theta \geq-0.40$ \\
$\hat{\theta}<-0.40$ & 0.4585 & 0.024 \\
$\hat{\theta} \geq-0.40$ & 0.040 & 0,4755 \\
\hline
\end{tabular}




\section{Capítulo 6}

\section{Conclusões}

Pode-se observar por meio dos resultados das simulações (baixo vício e erro quadrático médio para 20 ou 30 itens e estimaçao por EAP) e da aplicação real do TAI-PI que o teste adaptativo informatizado desenvolvido é viável para a avaliação da proficiência em língua inglesa dos alunos de pós-graduação do ICMC, além de possibilitar a compilação imediata do resultado do teste. Pelo nível educacional e formação dos examinados, não se esperava encontrar ninguém com dificuldade de realização do teste via computador. Isso se confirmou no dia das aplicações do TAI-PI e, portanto, supõe-se que a mudança do formato papel-e-caneta para informatizado não ocasiona nenhum confundimento relevante para a avaliação da proficiência em inglês. Os resultados obtidos evidenciam que um teste de 25 itens parece ser suficiente para estimar satisfatoriamente o traço latente em questão (Van der Linden e Pashley 2010). Com os estudos de classificação será possível determinar um ponto de corte para a proficiência do indivídio e em uma próxima aplicação poder usá-lo para classificar o aluno em aprovado ou reprovado. Portanto o TAI-PI poderá ser uma nova forma de avaliação a ser utilizada para os alunos da pós-graduação do ICMC, em que cada aluno terá uma prova individual e que melhor o represente. Como já evidenciado um TAI é uma forma mais eficiente de avaliar os alunos, o que irá acrescentar muito mais agilidade e confiabilidade do teste.

\subsection{Sugestões para Pesquisas Futuras}

Vários estudos devem ser realizados para o aperfeiçoamento do TAI-PI. Pode-se dividí-los e três aspectos: (i) o aprimoramento do banco de itens e da interpretação da escala, que é um aspecto de trabalho a ser realizado pelo especialista em língua inglesa, (ii) o aperfeiçoamento do software propriamente dito, que é tarefa do especialista em computação, e (iii) a validação do TAI-PI, tanto sob o aspecto de correta classificação do indivíduo em aprovado/reprovado, quanto sob o aspecto da metodologia estatística implementada. Este último, tarefa específica da área da estatística.

O aprimoramento do banco, será focado na revisão dos itens que tiveram estimativas dos parâmetros ruins (estimativas de a baixas, por exemplo). Além disso, itens novos serão criados, tanto na forma tradicional, quanto na forma interativa, ou seja, usando do fato do item ser aplicado via computador e viabilizar a exibição de imagem, som e interatividade com o examinado. Para esta tarefa, a pesquisadora Andrea Jéssica Borges Monzón, colaboradora deste projeto de pesquisa, contará com uma aluna bolsista de iniciação científica da área de Letras, especialidade em inglês. 


\title{
Referências Bibliográficas
}

\begin{abstract}
Aluísio et al.(2003) Sandra Aluísio, Valeria de Aquino, Rafael Pizzirani e Osvaldo de Oliveira. Assessing high-order skills with partial knowledge evaluation: Lessons learned from using a computer-based proficiency test of english for academic purposes. Journal of Information Technology Education: Research, 2(1):185-201. Citado na pág. 3
\end{abstract}

Baker(2001) F.B. Baker. The basic of Item Response Theory. EUA: ERIC Clearinghouse on Assessment and Evaluation. , 2 edição. Citado na pág. i, iii, 9

Baker e Kim(2004) Frank B Baker e Seock-Ho Kim. Item response theory: Parameter estimation techniques. CRC Press. Citado na pág. 9

Bloom(1984) Benjamin Bloom. Taxonomy of Educational Objectives. New York, Longman. Citado na pág. 3

Bridgeman e Cline(2000) Brent Bridgeman e Frederick Cline. Variations in mean response times for questions on the computer-adaptive gre $₫$ general test: Implications for fair assessment. ETS Research Report Series, 2000(1):i-29. Citado na pág. 2

Chang e Ying(1996) Hua-Hua Chang e Zhiliang Ying. A global information approach to computerized adaptive testing. Applied Psychological Measurement, 20(3):213-229. Citado na pág. 9

Conejo et al.(2001) Ricardo Conejo, Eva Millán, Jose-Luis Perez-de-la Cruz e Mónica Trella. Modelado del alumno: un enfoque bayesiano. Inteligencia Artificial, Revista Iberoamericana de Inteligencia Artificial, 5(12):50-58. Citado na pág. 11

de Andrade et al.(2000) Dalton Francisco de Andrade, Heliton Ribeiro Tavares e Raquel da Cunha Valle. Teoria da resposta ao item: conceitos e aplicações. ABE, Sao Paulo. Citado na pág. 17

Dehghan et al.(2006) Mehdi Dehghan, M Masjed-Jamei e MR Eslahchi. On numerical improvement of open newton-cotes quadrature rules. Applied mathematics and computation, 175(1): 618-627. Citado na pág. 9

Eignor et al.(1998) Daniel Eignor, Carol Taylor, Irwin Kirsch e Joan Jamieson. Development of a scale for assessing the level of computer familiarity of toefl examinees. ETS Research Report Series, 1998(1):i-32. Citado na pág. 2

Eignor(1993) Daniel R Eignor. Deriving comparable scores for computer adaptive and conventional tests: An example using the sat1, 2. ETS Research Report Series, 1993(2):i-16. Citado na pág. 2

Goncalves(2004) S. M.; Oliveira L. H. M. de ; Oliveira JUNIOR O. N. Goncalves, J. P.; Aluísio. A learning environment for english for academic purposes based on adaptive tests and task-based systems. Intelligent Tutoring Systems: 7th International Conference, ITS 2004, Maceió, Alagoas, Brazil, August 30-September 3, 2004, Proceedings, 175(1):1-11. Citado na pág. 4 
Kirsch et al.(1998) Irwin Kirsch, Joan Jamieson, Carol Taylor e Daniel Eignor. Computer familiarity among toefl examinees. ETS Research Report Series, 1998(1):i-23. Citado na pág. 2

Klinger(1997) Allen Klinger. Experimental validation of learning accomplishment. Em Frontiers in Education Conference, 1997. 27th Annual Conference. Teaching and Learning in an Era of Change. Proceedings., volume 3, páginas 1367-1372. IEEE. Citado na pág. 3

Lans(2006) Rick F Lans. Introduction to sql: mastering the relational database language. Citado na pág. 5

McNeil et al.(1975) Barbara J McNeil, Emmett Keeler e S James Adelstein. Primer on certain elements of medical decision making. New England Journal of Medicine, 293(5):211-215. Citado na pág. 25

Metz(1978) Charles E Metz. Basic principles of roc analysis. Em Seminars in nuclear medicine, volume 8, páginas 283-298. Elsevier. Citado na pág. 25

Mislevy(1986) Robert J Mislevy. Bayes modal estimation in item response models. Psychometrika, 51(2):177-195. Citado na pág. 13

Mislevy e Stocking(1989) Robert J Mislevy e Martha L Stocking. A consumer's guide to logist and bilog. Applied psychological measurement, 13(1):57-75. Citado na pág. 9

Monzón(2010) Andrea Jessica Borges Monzón. Construçao de banco de questoes para exames de proficiência em inglês para programas de pós-graduaçao. Citado na pág. 2

Piton-Gonçalves et al.(2009) Jean Piton-Gonçalves, Andrea Jessica Borges Monzón e Sandra Maria Aluísio. Métodos de avaliação informatizada que tratam o conhecimento parcial do aluno e geram provas individualizadas. Em Anais do Simpósio Brasileiro de Informática na Educação, volume 1. Citado na pág. 4

Ricarte(2013) Thales Akira Matsumoto Ricarte. Teste adaptativo computadorizado nas avaliações educacionais e psicológicas. Tese de Doutorado, Universidade de São Paulo. Citado na pág. 5

Rudner(2005) Lawrence M Rudner. Expected classification accuracy. Practical Assessment, Research É Evaluation, 10(13):1-4. Citado na pág. 26

Samejima(1969) Fumiko Samejima. Estimation of latent ability using a response pattern of graded scores. Psychometrika monograph supplement. Citado na pág. i, iii

Sands et al.(1997) William A Sands, Brian K Waters e James R McBride. Computerized adaptive testing: From inquiry to operation. American Psychological Association. Citado na pág. 2

Segall(1993) DO Segall. Score equating verification analyses of the cat-asvab. Briefing presented to the Defense Advisory Committee on Military Personnel Testing. Williamsburg, VA, USA. Citado na pág. 2

Shuford Jr et al.(1966) Emir H Shuford Jr, Arthur Albert e H Edward Massengill. Admissible probability measurement procedures. Psychometrika, 31(2):125-145. Citado na pág. i, iii

Sukamolson(2002) Suphat Sukamolson. Computerized test/item banking and computerized adaptive testing for teachers and lecturers. Information Technology and Universities in Asia-ITUA. Citado na pág. 11

Van der Linden(2000) Wim J Van der Linden. Constrained adaptive testing with shadow tests. Computerized adaptive testing: Theory and practice, páginas 27-52. Citado na pág. 10, 18

Van der Linden e Glas(2010) Wim J Van der Linden e Cees AW Glas. Elements of adaptive testing. Springer. Citado na pág. 11 
Van der Linden e Pashley(2010) Wim J Van der Linden e Peter J Pashley. Item selection and ability estimation in adaptive testing. Em Elements of adaptive testing, páginas 3-30. Springer. Citado na pág. i, iii, 11, 29

Wainer e Wang(2000) Howard Wainer e Xiaohui Wang. Using a new statistical model for testlets to score toefl. Journal of Educational Measurement, 37(3):203-220. Citado na pág. 2

Wainer et al.(2000) Howard Wainer, Neil J Dorans, Ronald Flaugher, Bert F Green e Robert J Mislevy. Computerized adaptive testing: A primer. Routledge. Citado na pág. 2

Weiss(1982) David J Weiss. Improving measurement quality and efficiency with adaptive testing. Applied Psychological Measurement, 6(4):473-492. Citado na pág. 1 